\title{
Online Algorithms for Factorization-Based Structure from Motion
}

\author{
Ryan Kennedy $^{\mathrm{a}, *}$, Laura Balzano ${ }^{\mathrm{b}}$, Stephen J. Wright ${ }^{\mathrm{c}}$, Camillo J. Taylor ${ }^{\mathrm{a}}$ \\ ${ }^{a}$ University of Pennsylvania \\ ${ }^{b}$ University of Michigan \\ ${ }^{c}$ University of Wisconsin-Madison
}

\begin{abstract}
We present a family of online algorithms for real-time factorization-based structure from motion, leveraging a relationship between the incremental singular value decomposition and recently proposed methods for online matrix completion. Our methods are orders of magnitude faster than previous state of the art, can handle missing data and a variable number of feature points, and are robust to noise and sparse outliers. We demonstrate our methods on both real and synthetic sequences and show that they perform well in both online and batch settings. We also provide an implementation that is able to produce 3D models in real time using a laptop with a webcam.
\end{abstract}

Keywords: structure from motion, matrix completion, incremental singular value decomposition

\section{Introduction}

The problem of structure from motion - recovering the 3D structure of an object and locations of the camera from a monocular video stream - has been studied extensively in computer vision. For the rigid case, which is the focus of this paper, many algorithms are based on the seminal work of Tomasi and Kanade 11. In this work it was shown that a noise-free measurement matrix of point tracks has rank at most 3 for an affine camera when the data are centered at the origin. The 3D locations of all tracked points and camera positions can be easily obtained from a factorization of this matrix. Due to occlusion, however, the matrix is typically missing many entries, so standard matrix factorization techniques cannot be applied.

Recent work in low-rank matrix completion has explored conditions under which the missing entries in a low-rank matrix can be determined, even when the matrix is corrupted with noise or sparse outliers [2, 3, 4, 5]. Most algorithms for matrix completion are static in nature, working with a "batch" of matrix columns. In this paper, we focus on online structure from motion, in which the 3D model of point locations must be updated in real time as the video is captured. The algorithm must be efficient enough to run in real time, yet still must deal effectively with missing data and noisy observations. The online problem has received little attention in comparison to batch algorithms, although online algorithms have been developed for matrix completion [6, 7] that have shown promise in other real-time computer vision applications [8]. In this paper, we extend these online algorithms to the problem of rigid structure from motion.

Our main contribution is a suite of online matrix completion algorithms that can be applied to the structure from motion (SFM) problem. The main algorithm SAGE is based on [6], but differs fundamentally in that it is based on matrix factorization instead of incremental gradient descent. Our other algorithms are variations on SAGE, and they inherit this property. Our algorithms address several difficulties that have been observed in this field, specifically: (1) our method is inherently online and provides a matrix estimate after every new update, (2) we are able to handle a dynamically changing number of features, (3) we directly deal with missing data, (4) we naturally deal with data that are offset from the origin, (5) our algorithms

${ }^{*}$ Corresponding author at: Department of Computer and Information Science, University of Pennsylvania, Philadelphia PA

Email address: kenry@cis. upenn.edu (Ryan Kennedy) 
can be made robust to outliers, and (6) our method is extremely fast. We note that (1) and (2) together imply that our algorithms can accommodate changes in the both the numbers of rows and columns.

In addition to testing with online data, we show that our algorithms are competitive with - and often orders of magnitude faster than - state-of-the-art batch algorithms. To demonstrate the utility of our approach, we describe a laptop implementation that creates 3D models of objects in real time using video from an attached webcam.

\section{Related Work}

\subsection{Structure From Motion}

Much research on the rigid structure-from-motion problem is based on Tomasi and Kanade's factorization algorithm [1] for orthographic cameras, and subsequent work 9] that extended its applicability to other camera models [10, 11, 12, 13, 14, 15. Comparatively little work has been done on online structure from motion, apart from algorithms that employ batch methods or local bundle adjustment in an online framework [16, 17].

In [14, Mortia and Kanade proposed a sequential version of the factorization algorithm, but their method cannot deal with missing data, nor can they handle outliers or a dynamically changing set of features. The approach of McLauchlan and Murray [18] can handle missing data but uses simplifying heuristics to achieve a low computational complexity. The related algorithm of Trajković and Hedley [19] dispenses with the heuristics, but focuses on tracking of moving objects within a scene. More recently, Cabral et al. [20] proposed a method that performs matrix completion iteratively, in an online manner. However, it is difficult to add new features dynamically with their approach, and it can be numerically unstable. The algorithm most similar to our own is the incremental SVD (ISVD) approach of Bunch and Nielsen [21, which has been previously adapted to handle missing data $[22,35$. In Section 4.2 we show how ISVD is related to our algorithms.

\subsection{Matrix Completion and Subspace Tracking}

Low-rank matrix completion is the problem of recovering a low-rank matrix from an incomplete sample of the entries. It was shown in [3, 23] that under assumptions on the number of observed entries and on incoherence of the singular vectors of this matrix with respect to the canonical coordinate axes, the nuclear norm minimization convex optimization problem solves the NP-hard rank minimization problem exactly. Since this breakthrough, a flurry of research activity has centered around developing faster algorithms to solve this convex optimization problem, both exactly and approximately; see [24, 25] for two examples. The algorithm GROUSE [6] (Grassmannian Rank-One Update Subspace Estimation) performs incremental gradient descent on a non-convex version of the matrix completion problem that admits fast online updates. GROUSE outperforms all non-parallel algorithms in computational efficiency, often by an order of magnitude, while remaining competitive in terms of estimation error when the noise is small.

The GROUSE algorithm was developed for low-dimensional subspace tracking with incomplete data. This is an area of extensive research. Comprehensive reference lists for complete-data adaptive methods for tracking subspaces and extreme singular values and vectors of covariance matrices can be found in [26, 27, where methods from the matrix computation literature and gradient-based methods from the signal processing literature are all discussed. Since GROUSE is an online method, it is a natural candidate for structure from motion, but it is not quite adequate in that it estimates only the column space of the matrix, and requires a final projection of all incomplete columns onto this column space to complete the matrix. Therefore, the unadorned form of GROUSE is not appropriate for contexts where (1) the number of rows is changing dynamically and (2) real-time completion is of interest - both of which are true in real-time structure from motion. Our algorithm SAGE (named after the sage grouse) addresses both these issues using a matrix factorization formulation.

A useful extension of these matrix completion algorithms is toward "Robust PCA," which seeks to recover a low-rank matrix in the presence of outliers [28, 29, 30, 31. This work has had application in computer vision, decomposing a scene from several video frames as the sum of a low-rank matrix of background (which 
represents the global appearance and illumination of the scene) and a sparse matrix of moving foreground objects [4, 32. GRASTA [8, 33] (Grassmannian Robust Adaptive Subspace Tracking Algorithm) is a robust extension of GROUSE that performs online estimation of a changing low-rank subspace while subtracting outliers. Our algorithm RSAGE is a robust version of SAGE that features a similar extension.

\subsection{Contributions of $S A G E$}

We now comment on the novelty of SAGE with respect to previous contributions. While the original ISVD algorithm provides a matrix estimate that is updated with each streaming column or added row, it does not naturally handle missing data or revisiting past points. While the GROUSE algorithm handles missing data, it does not update the row space simultaneously with the column space, so does not provide a full matrix estimate at each iteration. SAGE combines the benefits of the two approaches: It allows missing data and provides a matrix estimate at every iteration. Further, SAGE inherits the speed of GROUSE, making it orders of magnitude faster than most other algorithms on a variety of structure from motion problems, and allowing it to be used in truly real-time contexts.

The version of ISVD that is adapted to missing data [22, 35] has a drawback: It relies heavily on early subspace estimates, since the update incorporates singular value estimates. Therefore when there is a lot of missing data, early estimates of the subspace and singular values are poor and convergence is slow. By contrast, the GROUSE algorithm is agnostic to previous data; all directions of the current subspace carry equal weight. SAGE inherits this property of GROUSE.

Minor contributions of SAGE include the incorporation of the all-ones offset vector into the column space, the ability to revisit previous columns to improve the matrix estimate, and a variant that is robust to sparse outliers. Additionally, it is novel among matrix completion algorithms in its ability to handle added rows, to the best of our knowledge. This feature makes it potentially useful in several other applications, e.g., recommender systems, where both the number of users and number of products are changing over time.

In summary, this paper presents the first fully online factorization-based structure-from-motion algorithms, whose speed improvements over earlier techniques allow them to be applied in real-time settings.

\section{Problem Description}

Given a set of points tracked through a video, the measurement matrix $W$ is defined as

$$
W=\left[\begin{array}{ccc}
x_{1,1} & \ldots & x_{1, m} \\
\vdots & \ddots & \vdots \\
x_{n, 1} & \ldots & x_{n, m}
\end{array}\right],
$$

where $x_{i, j} \in \mathbb{R}^{1 \times 2}$ is the projection of point $i$ onto the camera at frame $j$, giving $W$ a size of $n \times 2 m$, where $n$ is the number of points and $m$ is the number of frames. If we assume that the data are centered at the origin, then in the absence of noise this matrix has rank at most 3 and can be factored as

$$
W=\tilde{S} \tilde{M}^{T},
$$

where $\tilde{S}$ is the $n \times 3$ structure matrix containing 3D point locations, while $\tilde{M}$ is the $2 m \times 3$ motion matrix, which is the set of affine camera matrices corresponding to each frame [1. Data that are offset from the origin have an additional translation vector $\tau$; we can write

$$
W=\left[\begin{array}{ll}
\tilde{S} & 1
\end{array}\right]\left[\begin{array}{ll}
\tilde{M} & \tau
\end{array}\right]^{T}=S M^{T},
$$

where $W$ now has rank at most 4 and the constant ones vector is necessarily part of its column space. We use this fact to naturally deal with offset data in our algorithms (Section 5.1).

In the presence of noise, one can use the singular value decomposition (SVD) [1] in order to find $S$ and $M$ such that $S M^{T}$ most closely approximates $W$ in either the Frobenius or operator norm. In this paper we address the problem of online structure from motion in which we have an estimated factorization at time $t$ 
- namely, $\hat{W}_{t}=\hat{S}_{t} \hat{M}_{t}^{T}$ - and we wish to update our estimate at time $t+1$ as we track points into the next video frame. The new information takes the form of two additional columns for $W_{t}$, leading to successive updates of the form $W_{t+1}=\left[\begin{array}{ll}W_{t} & v_{t}\end{array}\right]$. The algorithm for updating the approximate factorization must be efficient enough to be used in real time, able to handle missing data as points become occluded, and able to incorporate new points.

\section{Matrix Completion Algorithms for SFM}

\subsection{Background on the GROUSE Algorithm}

We start by reviewing the GROUSE algorithm and its application to structure from motion, in order to set the stage for our proposed algorithm SAGE and its variants, which are discussed in later subsections.

As in Section 3, we use the subscript $t$ to denote values at time $t$. Let $n_{t}$ be the number of tracks started up to time $t$, let $U_{t} \in \mathbb{R}^{n_{t} \times 4}$ be a matrix with orthonormal columns that span the rank-4 column space of the measurement matrix $W_{t}$, and let $v_{t}$ be a new column such that $W_{t+1}=\left[\begin{array}{ll}W_{t} & v_{t}\end{array}\right]$. (We consider the two new columns provided by each video frame one at a time.) The set of observed indices of $v_{t}$ is denoted by $\Omega_{t} \subseteq\left\{1, \ldots, n_{t}\right\}$, and $v_{\Omega_{t}}$ and $U_{\Omega_{t}}$ are row submatrices of $v_{t}$ and $U_{t}$ that correspond to $\Omega_{t}$.

The GROUSE algorithm [6, 7] measures the error between the current subspace-spanning matrix $U_{t}$ and the new vector $v_{t}$ using the squared- $\ell_{2}$ distance

$$
\mathcal{E}\left(U_{\Omega_{t}}, v_{\Omega_{t}}\right)=\left\|U_{\Omega_{t}} w_{t}-v_{\Omega_{t}}\right\|_{2}^{2}
$$

where1

$$
w_{t}=U_{\Omega_{t}}^{+} v_{\Omega_{t}}
$$

is the set of weights that project $v_{\Omega_{t}}$ orthogonally onto the subspace given by $U_{\Omega_{t}}$. We would like to replace $U_{t}$ by an updated matrix with orthonormal columns of the same dimensions, that reduces the error $\mathcal{E}$ in accordance with the new observation $v_{t}$. Denoting by $r_{t}$ the residual vector for the latest observation, we define the subvector corresponding to the indices in $\Omega_{t}$ by

$$
r_{\Omega_{t}}=v_{\Omega_{t}}-U_{\Omega_{t}} w_{t}
$$

and set the components of $r_{t}$ whose indices are not in $\Omega_{t}$ to zero. We can express the sensitivity of the error $\mathcal{E}$ to $U_{t}$ as follows:

$$
\frac{\partial \mathcal{E}}{\partial U_{t}}=-2 r_{t} w_{t}^{T}
$$

GROUSE essentially performs projected gradient descent on the Grassmann manifold, taking a step in the negative gradient direction along the Grassmann manifold to maintain orthonormality; see [6] for details.

In structure from motion it is known a priori that $\operatorname{rank}(W) \leq 4$, and so a subspace estimation algorithm like GROUSE, which requires the rank (or an upper bound) as an input parameter, seems applicable. However, there are several issues that prevent GROUSE from being easily used for online structure from motion. First, GROUSE only maintains an estimate of the column space of $W_{t}$, so the corresponding matrix $R_{t} \in \mathbb{R}^{2 m_{t} \times 4}$ for which $\hat{W}_{t}=U_{t} R_{t}^{T}$ must be computed whenever a final reconstruction is needed. This can be a problem for online applications since it requires keeping all data until the algorithm is complete. Additionally, the choice of a critical parameter in GROUSE - the step size for gradient descent - can affect the rate of convergence strongly. Both issues would be resolved in an algorithm based on matrix factorization, but standard algorithms of this class do not handle missing or streaming data. Our SAGE algorithm, discussed below, leverages the relationship of GROUSE and incremental SVD to resolve these difficulties.

\footnotetext{
${ }^{1}$ Our notation $A^{+} b$ denotes the least-residual-norm solution to the least-squares problem $\min _{x}\|A x-b\|_{2}^{2}$, obtainable from a singular value decomposition of $A$ or a factorization of $A^{T} A$.
} 


\subsection{The Missing Data Incremental SVD (MD-ISVD) Formulation}

The incremental SVD algorithm 21 is a simple method for computing the SVD of a collection of data by updating an initial decomposition one column at a time. Given a matrix $W_{t}$ of rank $k$ whose thin SVD is $W_{t}=U_{t} \Sigma_{t} V_{t}^{T}$, we wish to compute the SVD of a new matrix with a single column added: $W_{t+1}=\left[\begin{array}{ll}W_{t} & v_{t}\end{array}\right]$. Defining $w_{t}=U_{t}^{T} v_{t}$ and $r_{t}=v_{t}-U_{t} w_{t}$, we have

$$
W_{t+1}=\left[\begin{array}{ll}
U_{t} & \frac{r_{t}}{\left\|r_{t}\right\|}
\end{array}\right]\left[\begin{array}{cc}
\Sigma_{t} & w_{t} \\
0 & \left\|r_{t}\right\|
\end{array}\right]\left[\begin{array}{cc}
V_{t}^{T} & 0 \\
0 & 1
\end{array}\right]
$$

where one can verify that the left and right matrices still have orthonormal columns. An SVD of the center matrix yields

$$
\left[\begin{array}{cc}
\Sigma_{t} & w_{t} \\
0 & \left\|r_{t}\right\|
\end{array}\right]=\tilde{U} \tilde{\Sigma} \tilde{V}^{T}
$$

where $\tilde{U}, \tilde{\Sigma}$, and $\tilde{V}$ are of size $(k+1) \times(k+1)$.

Two changes to ISVD are needed to make it suitable for online matrix completion. First, to handle missing data, we define the weights and residual vector in these update formulae as in Equations (5) and (6), respectively. Second, ensure that $w_{t}$ and $r_{\Omega_{t}}$ are well defined by (5) and (6), we limit the rank to $k$ and compute the thin SVD [34]. Since only the first $k$ singular vectors are needed, the smallest singular value and its associated singular vectors can be dropped. Let $\hat{U}, \hat{\Sigma}$, and $\hat{V}$ be the $(k+1) \times k, k \times k$ and $(k+1) \times k$ matrices resulting from this process. The updated rank- $k$ SVD estimate is then given by $W_{t+1}=U_{t+1} \Sigma_{t+1} V_{t+1}^{T}$, where

$$
U_{t+1}=\left[\begin{array}{ll}
U_{t} & \frac{r_{t}}{\left\|r_{t}\right\|}
\end{array}\right] \hat{U} \quad ; \quad \Sigma_{t+1}=\hat{\Sigma} \quad ; \quad V_{t+1}=\left[\begin{array}{cc}
V_{t} & 0 \\
0 & 1
\end{array}\right] \hat{V} .
$$

We call the resulting algorithm MD-ISVD (for "missing-data ISVD"), and note that this algorithm has been previously proposed in 22, 35. Although this algorithm improves over ISVD by allowing missing data, it has been observed empirically [35] that the convergence of MD-ISVD is slow when even a modest fraction of the data is missing. The column-space update involves an estimate of the singular values $\Sigma_{t}$, and for larger singular values, the update will favor the corresponding columns of $U_{t}$, even though in early iterations those columns are poor estimates based on partial data. In [22, this issue is addressed by down-weighting the singular values with a forgetting factor $\beta<1$. SAGE instead addresses this issue by being agnostic to singular values and using an identity matrix in the update, as we show next.

\subsection{The SAGE Algorithm}

We now examine the relationship of this MD-ISVD update to the GROUSE update, in the context of SFM. Let $\hat{W}_{t}=U_{t} R_{t}^{T}$ be an estimated rank-4 factorization of $W_{t}$ such that $U_{t}$ has orthonormal columns. Given a new column $v_{t}$ with observed entries $\Omega_{t}$, if $w_{t}$ and $r_{t}$ are the least-squares weight and residual vector, respectively, defined with respect to the set of observed indices $\Omega_{t}$ as in Equations $(5)$ and $(6)$, then we can write

$$
\left[\begin{array}{ll}
U_{t} R_{t}^{T} & \tilde{v}_{t}
\end{array}\right]=\left[\begin{array}{ll}
U_{t} & \frac{r_{t}}{\left\|r_{t}\right\|}
\end{array}\right]\left[\begin{array}{cc}
I & w_{t} \\
0 & \left\|r_{t}\right\|
\end{array}\right]\left[\begin{array}{cc}
R_{t} & 0 \\
0 & 1
\end{array}\right]^{T},
$$

where the subvector of $\tilde{v}_{t} \in \mathbb{R}^{n_{t}}$ corresponding to $\Omega_{t}$ is set to $v_{\Omega_{t}}$ while the remaining entries are imputed as the inner product of $w_{t}$ and the rows $i \notin \Omega_{t}$ of $U_{t}$. Stated formally, letting $\left[\tilde{v}_{t}\right]_{i}$ refer to the $i^{t h}$ component of the vector $\tilde{v}_{t}$, and we have:

$$
\left[\tilde{v}_{t}\right]_{i}:=\left\{\begin{array}{cc}
{\left[v_{t}\right]_{i}} & i \in \Omega_{t} \\
{\left[U_{t} w_{t}\right]_{i}} & i \in \Omega_{t}^{C}
\end{array} .\right.
$$

Define the SVD of the center matrix in Equation (11) to be

$$
\left[\begin{array}{cc}
I & w_{t} \\
0 & \left\|r_{t}\right\|
\end{array}\right]=\tilde{U} \tilde{\Sigma} \tilde{V}^{T}
$$


Let $\hat{U}$ and $\hat{V}$ be the $5 \times 4$ matrices obtained by dropping the last columns of $\tilde{U}$ and $\tilde{V}$, respectively, corresponding to the smallest singular value of $\tilde{\Sigma}$. Similarly, let $\hat{\Sigma}$ be the $4 \times 4$ diagonal matrix obtained by dropping the last column and row from $\tilde{\Sigma}$.

In [35], it was shown that updating $U_{t}$ to

$$
U_{t+1}=\left[\begin{array}{ll}
U_{t} & \frac{r_{t}}{\left\|r_{t}\right\|}
\end{array}\right] \hat{U}
$$

(similarly to the first equation in Equation (10)) is equivalent to GROUSE for a particular data-dependent choice of step size for the gradient algorithm. We use this update as the basis for SAGE.

Additionally, combining Equations (11) and (12), we may update $R_{t}$ as follows:

$$
R_{t+1}=\left[\begin{array}{cc}
R_{t} & 0 \\
0 & 1
\end{array}\right] \hat{V} \hat{\Sigma} .
$$

The result is a new rank-4 factorization $\hat{W}_{t+1}=U_{t+1} R_{t+1}^{T}$. Both $U_{t}$ and $R_{t}$ can be maintained with every update, so the estimated $\hat{W}_{t}$ is available in real-time. With further modifications to handle the addition of rows to the matrix and the all-ones offset vector in the column space (see Section 5), we may apply this algorithm to real-time structure from motion. We call this algorithm "SAGE."

We now comment on the advantages of SAGE. First, by updating both $U$ and $R$ simultaneously, there is no need to calculate $R$ whenever reconstruction is needed. Instead, we keep a running estimate of both $U$ and $R$ so estimates of the motion and structure matrices can easily be produced at any point in time. While the ISVD algorithm also updates both matrices, it can not handle missing data, and while the GROUSE algorithm handles missing data, it can not update both $U$ and $R$ simultaneously. With SAGE we thus have a truly online factorization-based SFM approach, because we no longer need to store the entire observation matrix $W$ in order to solve for $R$. Keeping a subset of the data may still be useful, so that old data can be revisited and reused when there is time available in the computation. The amount of data stored for this purpose may be limited to a fixed amount to constrain the memory footprint. Second, this formulation uses an implicit step size, as it is a matrix factorization approach as opposed to a gradient descent approach. Therefore we no longer are required to specify a step size as in the original GROUSE formulation, although the residual vector can still be scaled to change the effective step size if needed.

\subsection{RSAGE for Robust SFM}

The GRASTA algorithm [8, 33] is an extension of GROUSE that is robust to outliers. Instead of minimizing the $\ell_{2}$ cost function given in Equation (4), GRASTA uses the robust $\ell_{1}$ cost function

$$
\mathcal{E}_{\text {grasta }}\left(U_{\Omega_{t}}, v_{\Omega_{t}}\right)=\min _{w_{t}}\left\|U_{\Omega_{t}} w_{t}-v_{\Omega_{t}}\right\|_{1} .
$$

GRASTA estimates the weights $w_{t}$ of this $\ell_{1}$ projection as well as the sparse additive component using ADMM [36, then updates $U_{t}$ using Grassmannian geodesics, replacing $r_{t}$ with a variable $\Gamma_{t}$, which is a function of the sparse component and the $\ell_{1}$ weights [8]. We can use these $w_{t}$ and $\Gamma_{t}$ in place of $w_{t}$ and $r_{t}$ in SAGE (Section 4.2), resulting in a robust variant we call RSAGE. The novelty in RSAGE is to incorporate these $\ell_{1}$ weights and residuals into a matrix factorization framework. In Section 6, we show that RSAGE is better able to find the correct 3D structure in the presence of sparse outliers. While outside the scope of this paper, we believe this merging of ADMM-computed weights and residuals with a matrix factorization algorithm is worthy of more extensive study.

\subsection{SAGE, RSAGE, and MD-ISVD: A Family of Algorithms}

The algorithms that we have presented so far make up a family of algorithms that can be applied to SFM in various circumstances. We study SAGE and RSAGE along with MD-ISVD, which carries forward an estimate for the singular values, $\Sigma$. 
Another variant within this family could be obtained by scaling the residual norm in 12 by some value $\alpha_{t}$ before taking its SVD:

$$
\left[\begin{array}{cc}
I & w_{t} \\
0 & \alpha_{t}\left\|r_{t}\right\|
\end{array}\right]=\hat{U} \hat{\Sigma} \hat{V}^{T}
$$

This scaling potentially yields more control over the contribution of the residual vector to the new subspace estimate. We tried decreasing the value $\alpha_{t}$ with increasing $t$, and found this approach to be useful the batch setting. However, for online data, the no-scaling choice $\left(\alpha_{t}=1\right)$ performs best.

After we describe another relevant algorithmic component in the next section, the incorporation of an offset vector into the column space, we present in Algorithm 1 one iteration of each algorithm in the family discussed in this section. Table 1 shows the specifics of all proposed algorithms relative to this metaalgorithm.

\section{Implementation}

\subsection{The All-1s Vector}

A few more issues need to be addressed for implementation of SAGE for SFM. The first issue is to exploit the fact that the constant vector of ones (denoted as $\mathbb{1}$ ) should always be in the column space of $W_{t}$ and thus should be in the span of any estimate $U_{t}$ of the column space of $W_{t}$, since the points may be offset from the origin. Without loss of generality, suppose that $U_{t}$ has the ones-vector as its last column (appropriately scaled), so that

$$
U_{t}=\left[\begin{array}{ll}
\bar{U}_{t} & \mathbb{1} / \sqrt{n_{t}}
\end{array}\right], \quad R_{t}=\left[\begin{array}{ll}
\bar{R}_{t} & \tau_{t} \sqrt{n_{t}}
\end{array}\right] .
$$

Here $\tau_{t}$ is the corresponding translation vector, which is the (scaled) last column of $R_{t}$. Similarly, let $w_{t}=\left[\begin{array}{ll}\bar{w}_{t}^{T} & \gamma_{t}\end{array}\right]^{T}$. The derivative of the error $\mathcal{E}$ in Equation (4) with respect to just the first three columns is

$$
\frac{\partial \mathcal{E}}{\partial \bar{U}_{t}}=-2 r_{t} \bar{w}_{t}^{T}
$$

(cf. (7)). By not considering the derivative of $\mathcal{E}$ with respect to the ones vector it will remain in the span of $U$, since the SAGE update will be applied only to $\tilde{U}$. Our SAGE update for structure from motion is obtained by first setting

$$
\bar{U}_{t+1}=\left[\begin{array}{ll}
\bar{U}_{t} & \frac{r_{t}}{\left\|r_{t}\right\|}
\end{array}\right] \tilde{U} \quad ; \quad \bar{R}_{t+1}=\left[\begin{array}{cc}
\bar{R}_{t} & 0 \\
0 & 1
\end{array}\right] \tilde{V} \tilde{\Sigma} \quad ; \quad \tau_{t+1}=\left[\begin{array}{c}
\tau_{t} \\
\gamma_{t} / \sqrt{n_{t}}
\end{array}\right]
$$

(where $\tilde{U}, \tilde{\Sigma}$, and $\tilde{V}$ are defined as in Equation (12) using $\bar{w}_{t}$ ), and then dropping the last column of $\bar{U}_{t+1}$ and $\bar{R}_{t+1}$. Because the residual vector $r_{t}$ is still based on the full matrix $U_{t}$, including the ones-vector, $r_{t}$ will necessarily be orthogonal to the ones-vector. Therefore, since

$$
U_{t+1}=\left[\begin{array}{ll}
\bar{U}_{t+1} & \mathbb{1} / \sqrt{n_{t}}
\end{array}\right],
$$

$U_{t+1}$ will retain the ones-vector in its span and will still have orthonormal columns.

The same process can be applied to MD-ISVD. However, we note that the resulting method is no longer a true SVD, in that the updated $R_{t+1}$ will not necessarily have orthonormal columns. (In particular, the last column $\tau_{t}$ may not be orthogonal to the other columns, which make up the submatrix $\bar{R}_{t+1}$.) In our experiments, all algorithms are constrained to maintain the all-1's vector in their column space. Although this is no longer a true SVD, we found that it leads to better solutions than simply finding a rank-4 approximation. This observation has been reported previously, for example, in [11, where the best rank-4 factorization of the Dinosaur dataset was found to produce a suboptimal 3D model. 


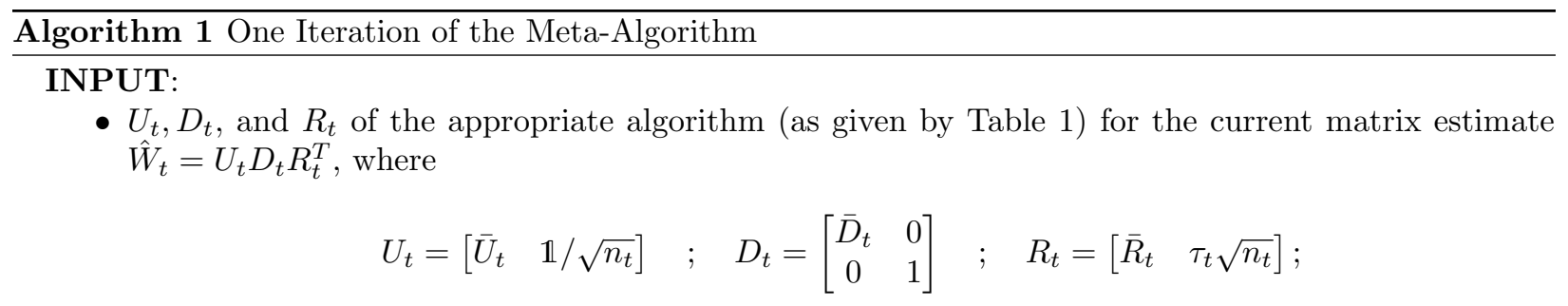

- scaling parameter $\alpha_{t}$;

- the new column $v_{\Omega_{t}}$ with only points indexed by $\Omega_{t}$ observed.

Calculate the weights $w_{t}=\left[\begin{array}{ll}\bar{w}_{t} & \gamma_{t}\end{array}\right]^{T}$ and residual $r_{t}$ for the appropriate algorithm according to Table 1 ;

Define $\bar{v}_{t}=\bar{U}_{t} \bar{w}_{t}+r_{t}$ to be the portion of the imputed vector $v_{t}=U_{t} w_{t}+r_{t}$ orthogonal to $\mathbb{1}$;

Noting that

$$
\left[\bar{U}_{t} \bar{D}_{t} \bar{R}_{t}^{T} \quad \bar{v}_{t}\right]=\left[\begin{array}{cc}
\bar{U}_{t} & \frac{r_{t}}{\left\|r_{t}\right\|}
\end{array}\right]\left[\begin{array}{cc}
\bar{D}_{t} & \bar{w}_{t} \\
0 & \left\|r_{t}\right\|
\end{array}\right]\left[\begin{array}{cc}
\bar{R}_{t} & 0 \\
0 & 1
\end{array}\right]^{T}
$$

compute the SVD of the update matrix (with scaled residual):

$$
\left[\begin{array}{cc}
\bar{D}_{t} & \bar{w}_{t} \\
0 & \alpha_{t}\left\|r_{t}\right\|
\end{array}\right]=\tilde{U}_{t} \tilde{\Sigma}_{t} \tilde{V}_{t}^{T}
$$

Update the column space and translation vector:

$$
\bar{U}_{t+1}=\left[\begin{array}{ll}
\bar{U}_{t} & \frac{r_{t}}{\left\|r_{t}\right\|}
\end{array}\right] \tilde{U}_{t} \quad ; \quad \tau_{t+1}=\left[\begin{array}{c}
\tau_{t} \\
\gamma_{t} / \sqrt{n_{t}}
\end{array}\right] ;
$$

Update $\bar{R}_{t+1}$ and $\bar{D}_{t+1}$ for the appropriate algorithm according to Table 1 ;

Drop the last column of both $\bar{U}_{t+1}$ and $\bar{R}_{t+1}$. Drop the last column and the last row of $\bar{D}_{t+1}$; Update

$$
U_{t+1}=\left[\begin{array}{lll}
\bar{U}_{t+1} & \mathbb{1} / \sqrt{n_{t}}
\end{array}\right] \quad ; \quad D_{t+1}=\left[\begin{array}{cc}
\bar{D}_{t+1} & 0 \\
0 & 1
\end{array}\right] \quad ; \quad R_{t+1}=\left[\begin{array}{ll}
\bar{R}_{t+1} & \tau_{t+1} \sqrt{n_{t}}
\end{array}\right]
$$

OUTPUT: $\hat{W}_{t+1}=U_{t+1} D_{t+1} R_{t+1}^{T}$.

\subsection{Adding New Points}

In online structure from motion, we are initially unaware of the total number of points to be tracked and need to account for newly added points as the video progresses. If $U_{t} \in \mathbb{R}^{n_{t} \times 4}$ is the current subspace estimate, then new points will manifest themselves as additional rows of $U_{t}$. However, when updating $U_{t}$, we have to make sure that the columns of $U_{t}$ remain orthonormal and the last column continues to be the vector of ones. We thus perform the following update when each new point is added, where we increment $n_{t+1}=n_{t}+1$ so that the columns of $U_{t}$ remain orthonormal:

$$
U_{t} \leftarrow\left[\begin{array}{cc}
\bar{U}_{t} & \mathbb{1} / \sqrt{n_{t}+1} \\
0 & 1 / \sqrt{n_{t}+1}
\end{array}\right] .
$$

We also update $\tau_{t}$, the last column of $R$, so that their product remains the same for current entries:

$$
\tau_{t} \leftarrow \tau_{t} \sqrt{\frac{n_{t}+1}{n_{t}}} .
$$




\begin{tabular}{|c|c|c|c|}
\hline \multicolumn{4}{|c|}{ A Family of Matrix Completion SFM Algorithms } \\
\hline & SAGE $\left(\ell_{2}\right)$ & RSAGE $\left(\ell_{1}\right)$ & MD-ISVD \\
\hline \multicolumn{4}{|l|}{ Matrix information } \\
\hline $\bar{D}_{t}=$ & $I_{k-1}$ & same as SAGE & $\Sigma_{t}$, an estimate of singular values \\
\hline $\bar{R}_{t}$ orthogonal? & no & no & yes \\
\hline \multicolumn{4}{|l|}{ For Algorithm 1} \\
\hline$w_{t}, r_{t}$ & $\begin{array}{l}w_{t}=\arg \min _{w}\left\|U_{\Omega_{t}} w-v_{\Omega_{t}}\right\|_{2}^{2} \\
r_{\Omega_{t}}=v_{\Omega_{t}}-U_{\Omega_{t}} w_{t} ; r_{\Omega_{t}^{C}}=0\end{array}$ & $\begin{array}{c}\text { computed using } \\
\text { ADMM (Section } 4.4\end{array}$ & $\begin{array}{l}\text { either SAGE or RSAGE } \\
\text { depending on cost }\end{array}$ \\
\hline Update $\bar{R}_{t+1}$ & $\bar{R}_{t+1}=\left[\begin{array}{cc}\bar{R}_{t} & 0 \\
0 & 1\end{array}\right] \tilde{V}_{t} \tilde{\Sigma}_{t}$ & same as SAGE & $\bar{R}_{t+1}=\left[\begin{array}{cc}\bar{R}_{t} & 0 \\
0 & 1\end{array}\right] \tilde{V}_{t}$ \\
\hline Update $\bar{D}_{t+1}$ & $\bar{D}_{t+1}=I_{k-1}$ & same as SAGE & $\bar{D}_{t+1}=\tilde{\Sigma}_{t}$ \\
\hline \multicolumn{4}{|l|}{ For Algorithm 2 } \\
\hline Initialization restrictions & 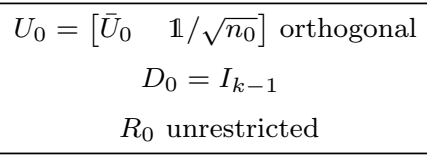 & same as SAGE & 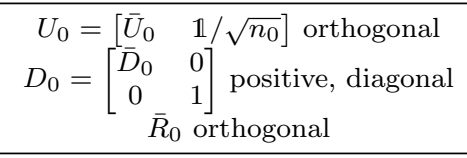 \\
\hline Remove a row of $R_{t}$ & just remove it & same as SAGE & downdate as in [34], then remove it \\
\hline
\end{tabular}

Table 1: Algorithmic specifics in each of our family of matrix completion algorithms for structure from motion, as used in Algorithm 1 and 2

\subsection{Updating Past Points}

The SAGE update for structure from motion is fast enough that many updates can be done for each new video frame. It is therefore advantageous to be able to revisit old frames and reduce the error more than would be possible using a single pass over the frames. Using the original SAGE formulation described in Section 4.1. we simply run additional SAGE updates using past columns of $W$. This does not work in the new online formulation, where we also keep track of the matrix $R_{t}$, since running another SAGE update will add a new row to $R_{t}$. Instead, we simply drop the associated row of $R_{t}$ before the update and replace it with the resulting new row. Because we do not impose any orthogonality restrictions on the matrix $R_{t}$, no correction is needed. In our experiments, however, we also compare to MD-ISVD, which requires that the the right-side matrix be orthogonal. (We "downdate" our SVD using the algorithm given by Brand 34 ] before performing an update.)

Having discussed these implementation details, we can define the outer loop of our algorithm, given in Algorithm 2, This outer loop calls Algorithm 1 to perform each iteration.

\subsection{Complexity}

Finally we comment on the per-iteration computational complexity of SAGE, RSAGE, and MD-ISVD. Recall that at iteration $t, n_{t}$ is the number of features, $T$ is the number of frames so far, $\left|\Omega_{t}\right|$ is the number of observations in the frame being used for the update, and $k$ is the rank of the decomposition. The computation of the weights in Algorithm 1 is the only place where the three algorithms differ. For SAGE and MD-ISVD, we must solve a Least Squares problem for the weights, giving $O\left(\left|\Omega_{t}\right| k^{2}\right)$ operations. For RSAGE, we use ADMM to compute the weights. Assuming that ADMM uses a constant number of iterations, this computation requires $O\left(\left|\Omega_{t}\right| k^{3}\right)$ operations. Computing the residual then requires $O\left(n_{t} k+\left|\Omega_{t}\right|\right)$ operations. To compute the SVD of the center $k \times k$ matrix we need $O\left(k^{3}\right)$ operations, and then updating $U$ and $R$ requires $O\left(n_{t} k^{2}\right)$ and $O\left(T k^{2}\right)$ operations respectively. In the typical situation where $k<\left|\Omega_{t}\right| \ll n_{t}$, these final two computations are the most burdensome, giving an overall computational complexity of $O\left(\left(n_{t}+T\right) k^{2}\right)$ for the $t^{\text {th }}$ iteration.

We emphasize that this is only the per iteration complexity. For these algorithms, we have yet to analyze how many iterations are required for convergence. While SAGE and MD-ISVD have the same per- 


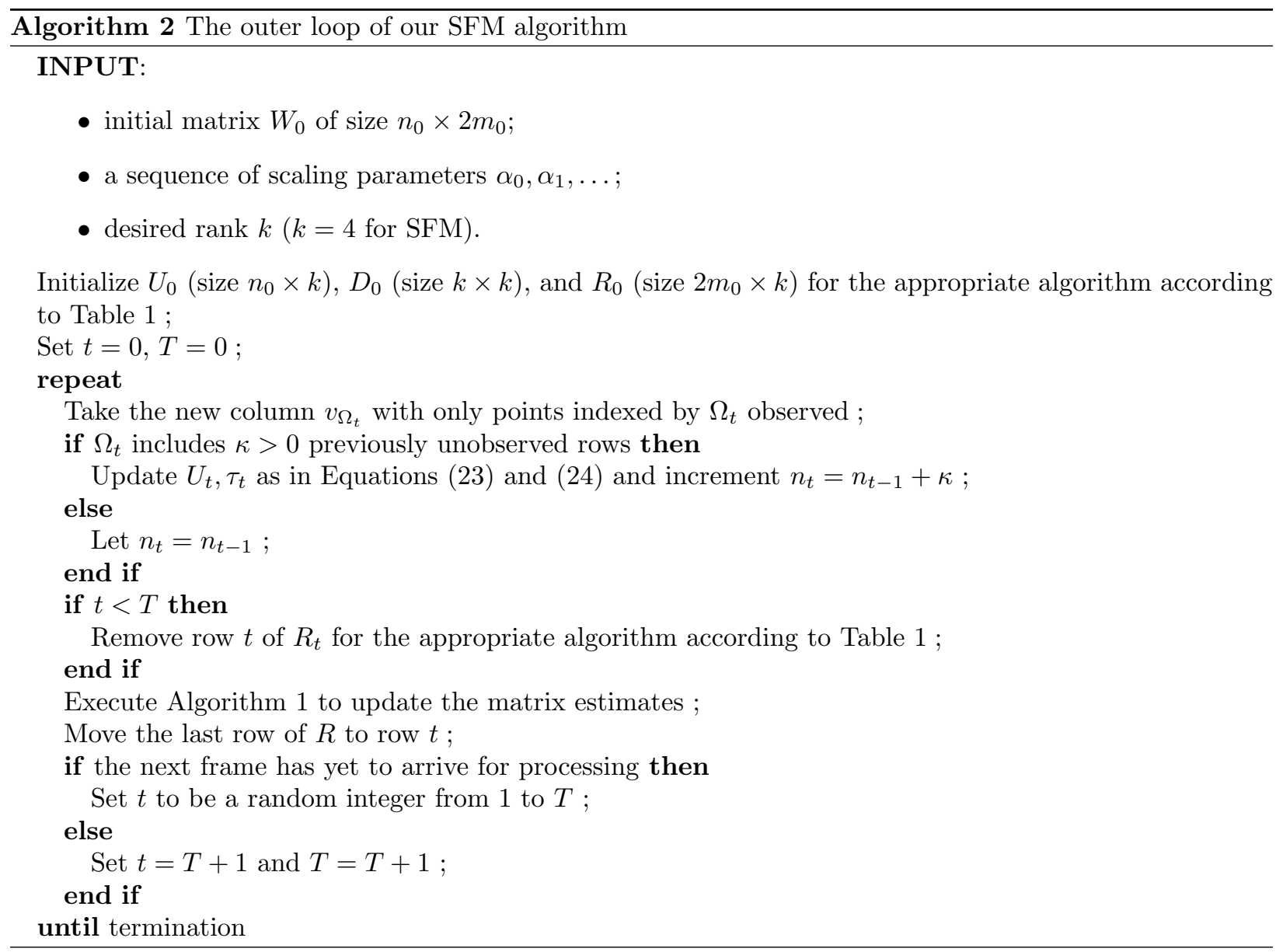

iteration complexity, they converge at very different speeds. Additionally, typical batch algorithms handle the whole matrix in every iteration, so the per-iteration complexity of SAGE is not directly comparable to the complexity given, e.g., in [37. Therefore, to show the practical impact on realtime structure from motion, we focus on runtime in our experiments.

\section{Experiments}

In this section we evaluate SAGE for rigid structure from motion in both the online and batch settings.

\subsection{Batch experiments}

\subsubsection{Algorithms}

Each of the algorithms we compare to was modified such that the constant ones-vector always remains in the column space of the estimated matrix. The algorithms we compare are:

- SAGE (SAGE and SAGE100) [6]: The main algorithm presented in this paper. In the batch setting, one iteration is defined as one pass over all columns of the measurement matrix in a random order. In SAGE, the residuals are not manually scaled. In SAGE100, we add an additional scaling of the residual in order to ensure convergence. The residual was scaled by $\alpha_{t}=C /(C+t)$ at iteration $t$, where $C=100$.

- Power Factorization (PF) [38]: Alternating least-squares optimization of $U$ and $R$. 
- Guerreiro and Aguiar (GA) [13: Iteratively fill in the missing matrix values using the current matrix estimate, and then find the best low-rank matrix with respect to this filled-in matrix.

- Damped Newton (DN) [11]: A second-order damped Newton's method with respect to all values of $U$ and $R$.

- Levenberg-Marquardt Subspace (LM_S) [37]: The cost function is redefined with respect to only $R$, and Levenberg-Marquardt minimization is used.

- Levenberg-Marquardt Manifold (LM_M) 37]: Similar to LM_S, but optimization is restricted to remain on the Grassmann manifold.

- Wiberg (WIBERG) [39]: A second-order method that linearizes the cost function around $U$ and performs a Newton-type minimization with respect to $R$.

- Damped Wiberg (DW) [40: A related method to the previous, this implementation adds a damping factor that allows for faster runtimes.

- Missing Data Incremental SVD (MD-ISVD) 22, 35]: As presented in this paper, it is similar to SAGE but additionally keeps track of the singular values. At each iteration, the current column's data is "downdated" before being subsequently updated.

- Column Space Fitting (CSF) [12]: A Levenberg-Marquardt optimization method that encourages the matrices of camera parameters to vary smoothly by restricting them to be composed of a basis of vectors in the discrete cosine transform domain. Based on the default parameters used in the code and the notes in the paper, we began with a set of basis vectors that is $10 \%$ of the total number of frames, and the basis is enlarged by this same amount every 100 iterations until the maximum size is reached.

- Bilinear Modeling via Augmented Lagrange Multipliers (BALM) 41]: A model which restricts the camera parameter matrices to lie on a particular manifold by projecting it onto the manifold after each iteration. Here, we use the scaled-orthographic projection. We update the Lagrange multipliers every 500 iterations. We found this number of iterations to perform well on most datasets, but note that better convergence may be obtained by fine-tuning this parameter to each dataset. For testing convergence, one iteration of BALM was defined as 50 internal iterations of the inner loop.

All algorithms were implemented within a common framework with a similar amount of optimization. All code is implemented using MATLAB, aside from one internal function for DN which is written in $\mathrm{C}++$. The experiments were run on Amazon EC2 computers with $1.8 \mathrm{GHz}$ Intel Core i5 processors and $4 \mathrm{~Gb}$ of RAM.

Note that CSF and BALM optimize somewhat different cost functions than the other algorithms, including SAGE. BALM has an additional constraint requiring the camera matrices to lie on a certain manifold. CSF uses a set of basis functions that assume the camera moves smoothly over time. All other algorithms attempt only to find a rank-4 factorization that minimizes the squared error of the observed entries, contingent on the constant ones vector being part of the factorization's column space. These algorithms do not impose constraints on the camera matrices themselves other than their rank, and thus they assume a general affine camera model. Only after a rank-4 solution is obtained do we impose scaled-orthographic metric constraints 9 to find a resulting 3D structure. We note that it is possible to incorporate additional constraints into SAGE. For example, just as is done in BALM, the solution could be projected onto an appropriate manifold after each iteration of SAGE, to ensure that the camera matrices are of the proper form. However, we did not encounter issues with degeneracy in our experiments, so did not find it necessary to implement this variant.

We imposed the same constraint on all rank-4 factorizations to include the all-ones vector. Note that several of the algorithms [40, 11, 37] previously report results that instead use general rank-4 factorizations without imposing the all-ones vector and subsequently measure only 2D RMSE. While this did not greatly affect convergence time, we found that the inclusion of the all-ones vector constraint may cause algorithms 


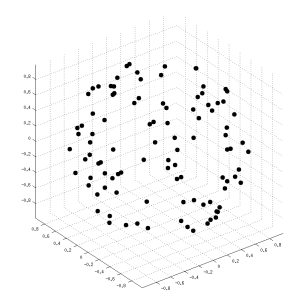

(a)

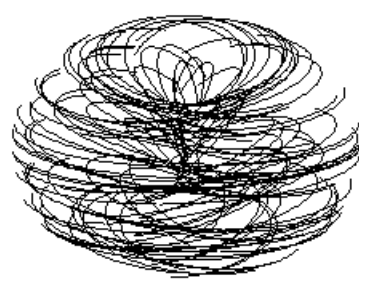

(b)

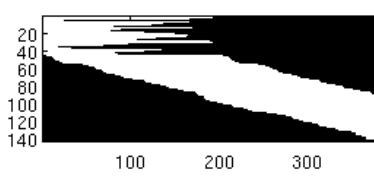

(c)

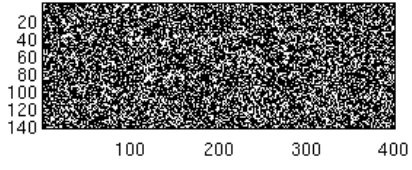

(d)

Figure 1: Synthetic Sphere dataset. (a) 3D model of the sphere. (b) Observed point tracks. (c) Observed data matrix based on occlusion pattern. (d) Observed data matrix with data removed randomly.

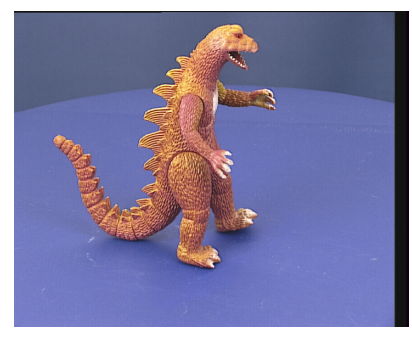

(a)

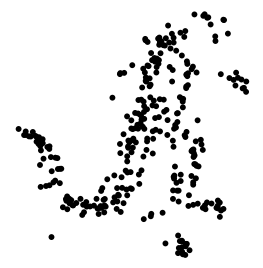

(b)

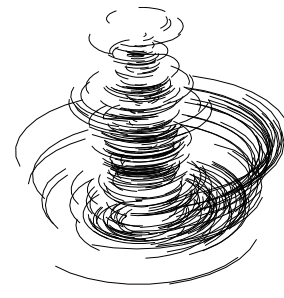

(c)

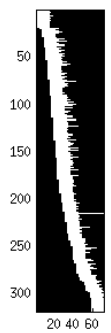

(d)

Figure 2: Dinosaur dataset. (a) One frame from the sequence. (b) Reconstructed 3D model. (c) Observed point tracks. Because the dinosaur is on a turntable, the tracks are elliptical. (d) Observed data matrix.

to get suck in local minima on some datasets (in terms of 2D RMSE). However, if the all-ones vector is not enforced, it would have to be enforced after convergence in order to obtain the final 3D model. A more in-depth comparison of the convegence of algorithms under different such constraints is worthy of further research.

All algorithms were run until convergence from 100 different random initializations. For CSF, a "random" initialization is not as straightforward because of its basis functions, and we initialized it by using it to approximate the random matrix and then using the resulting parameters as its initialization. We also evaluated each method using a deterministic initialization where values were filled in using the mean value of the corresponding column. For CSF, the deterministic initialization consists of using the default initialization as described in 12 .

We declared convergence to have occurred when the 2D RMSE did not decrease more than $1 \%$ in the previous 10 minutes or 10 iterations. For the synthetic dataset, we also measure 3D RMSE. These 3D reconstructions were created by applying standard scaled-orthographic metric constraints 9] and aligning them to the groundtruth model using a Procrustes transformation.

Note that while the datasets used here are captured with standard projective cameras, the factorization model we use assumes an affine camera. However, we have chosen datasets that have minimal perspective distortions, some of which have been previously used in factorization-based algorithms [42, 15. For videos that contain significant perspective distortions, other methods might be more appropriate. 


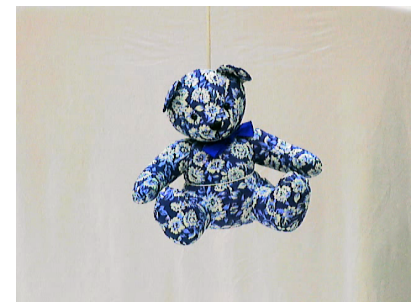

(a)

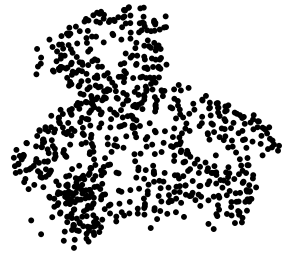

(b)

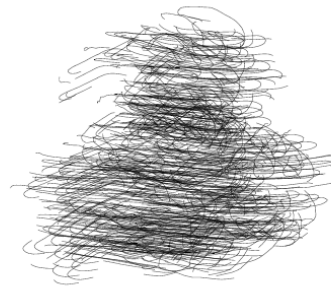

(c)

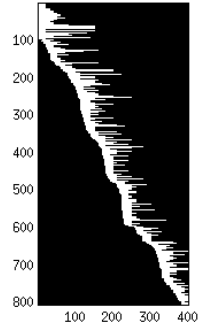

(d)

Figure 3: Bear dataset. (a) One frame from the sequence. (b) Reconstructed 3D model. (c) Observed point tracks. (d) Observed data matix.

\subsubsection{Datasets}

Three datasets are used in our experiments: Synthetic Sphere (Figure 11), Dinosaur [42] (Figure 2), and Bear [15] (Figure 3).

The Synthetic Sphere dataset is a 3D unit sphere with 100 randomly-placed points tracked over 200 frames. The camera was made to move in a smooth trajectory around the sphere and the data were projected onto the camera using an orthographic projection, so the problem has an exact rank-4 solution. In this case, points that became occluded and re-appeared were treated as two separate points and the resulting matrix is of size $143 \times 400$. We used two different data models for this dataset. In the first, occluded data points were removed from the data matrix, resulting in $65.1 \%$ missing data with a banded structure to the data matrix. In the second, we removed the same number of data points uniformly at random.

The Dinosaur ("Dino") dataset consists of 319 points over 36 frames, with $76.9 \%$ missing data. The Bear dataset has 806 points and 200 frames, with $88.6 \%$ missing data.

The Synthetic Dino dataset was generated from the Dino sequence as follows. The 3D model of the best reconstruction of the Dino was used as the ground truth, and was projected onto 100 random orthographic cameras, resulting in a matrix of size $319 \times 200.90 \%$ of the data were then removed uniformly at random.

\subsubsection{Results for the Synthetic Sphere}

Banded Occlusion Pattern We begin with the Synthetic Sphere dataset with a realistic occlusion pattern. The results of measuring the 2D RMSE for this dataset are shown in Figure 4 . The top set of plots show the convergence of each algorithm over time. We plot all 100 randomly-initialized runs in a lighter color, and the median run is plotted in a darker version of the same color. The dashed black line shows the run using the deterministic initialization. To measure the speed of convergence, we find the time it takes for the median line to move $99 \%$ of the way from its initial error to the smallest error achieved over all plots. The algorithms are sorted by this value and its location is denoted by a vertical red line. The bottom set of plots shows the same results when 3D RMSE is calculated. The set of plots in Figure 5 show the empirical cumulative distribution function of the error for each algorithm after convergence. Once again, the error for the deterministic initialization is shown with a black dashed line. In this case, the number associated with each algorithm is the median error after convergence and the algorithms are sorted by this value.

In terms of speed, we find that SAGE and SAGE100 are significantly faster than any other algorithm for 2D RMSE. These two algorithms take only 0.35 seconds to achieve $99 \%$ of the final error reduction. The only algorithm with comparable performance is $\mathrm{PF}$, which takes 10 times longer and does not converge as frequently. All other algorithms are at least two orders of magnitude slower than SAGE. It is also notable that the deterministic solution often performs as good as the best random initialization, with the exceptions being DW and DN. A similar result is seen with 3D RMSE in the middle plots, where PF and SAGE converge much faster than other algorithms. The general convergence rate in terms of $3 \mathrm{D}$ error is lower than $2 \mathrm{D}$ error, 
since the $3 \mathrm{D}$ error will generally not decrease much until the $2 \mathrm{D}$ error is lowered enough for an accurate $3 \mathrm{D}$ model to be generated.

It is important to note here that while SAGE reaches a small error in computation times that are orders of magnitude faster than the other algorithms, it is not always fastest to reach a very small error. Consider the time it takes to reach $10^{-2}$ versus $10^{-5}$ for $2 \mathrm{D}$ RMSE in this experiment. While SAGE reaches $10^{-2}$ in a median time of 0.26 seconds, it takes an additional 26.9 seconds to reach $10^{-5}$. Damped Wiberg (DW), by comparison, reaches $10^{-2}$ in 25.1 seconds and takes only another 9.52 seconds to reach $10^{-5}$. Thus, while our first-order method is faster at first, second-order methods have potential to reach a highly accurate solution, given more computation time. Since our focus is real-time structure from motion, we believe that SAGE is an excellent option for achieving aceptable accuracy in significantly less time.

With respect to convergence as shown in the bottom set of plots, we find that LM_M and LM_S almost always converge, regardless of their initialization; the same can be said for DW if we ignore the deterministic initialization. Other algorithms, including SAGE and WIBERG, also converge in most cases. Interestingly, the more complex algorithms CSF and BALM will often get stuck in local minima on this dataset. We note that these algorithms have several extra parameters that affect their convergence rate and it may be possible to tune them to specific datasets to get better convergence.

Random occlusion pattern. Results for the Synthetic Sphere dataset using a random pattern of missing data are shown in Figure 6. This dataset is much simpler for all algorithms; $100 \%$ convergence is achieved for all initializations. We omit 2D errors since they are nearly identical to the 3D error shown here. We omit the CDF plots too, since convergence is universally achieved. Again, we find that SAGE, SAGE100 and $\mathrm{PF}$ are several orders of magnitude faster than most other algorithms. For this dataset, these three algorithms converge to the groundtruth 3D sphere in less than a tenth of a second. Interestingly, in this case there is no accuracy trade-off; SAGE reaches an error as low as the best algorithm in orders of magnitude less computation time.

\subsubsection{Results for the Dino and Bear}

Results for the Dino and Bear datasets are shown in Figures 7 and 8 , respectively. For both datasets, the results are relatively similar. We find that SAGE100 and SAGE are again much faster than all other algorithms, with the scaled version SAGE100 being moderately faster than the unscaled SAGE. In terms of convergence, we again see that the second-order methods LM_S and LM_M converge nearly every time, with WIBERG also performing well. We also find the SAGE100 converges more often than the unscaled SAGE. For the Bear dataset, both CSF and BALM exhibit slow convergence, because the dataset is larger. We may

have observed more frequent convergence if we had allowed more computation time, but our upper limit of $10^{4}$ seconds is a generous time limit, and if the algorithm can not solve the problem in this time, it is worth looking for alternatives.

The trade-off between accuracy and computation time is even more prominent in these real datasets. Several algorithms converge to a solution that is not as accurate as the most accurate of the slower methods. One conclusion we draw is that if an accurate batch solution is required, it may be beneficial to combine SAGE100 with a second order method, in a two-phase strategy. SAGE100 would achieve a moderately accurate solution quickly in the first phase, while the second order method finds a highly accurate final solution in the second phase.

\subsubsection{Robust algorithms}

We compare SAGE100 with its robust counterpart RSAGE100, with results shown in Figure 9. We used the Synthetic Sphere dataset with a banded occlusion pattern. A varying proportion of the visible entries - from $0 \%$ to $35 \%$ - were set to sparse outliers in the range $[-100,100]$. Both SAGE100 and RSAGE100 were initialized using a deterministic initialization by filling in missing values with the mean of all values present in each column.

With even a small number of sparse outliers, SAGE is unable to find the correct 3D model. RSAGE handles the sparse outliers much better, with the 3D RMSE decaying gradually as the proportion of sparse 

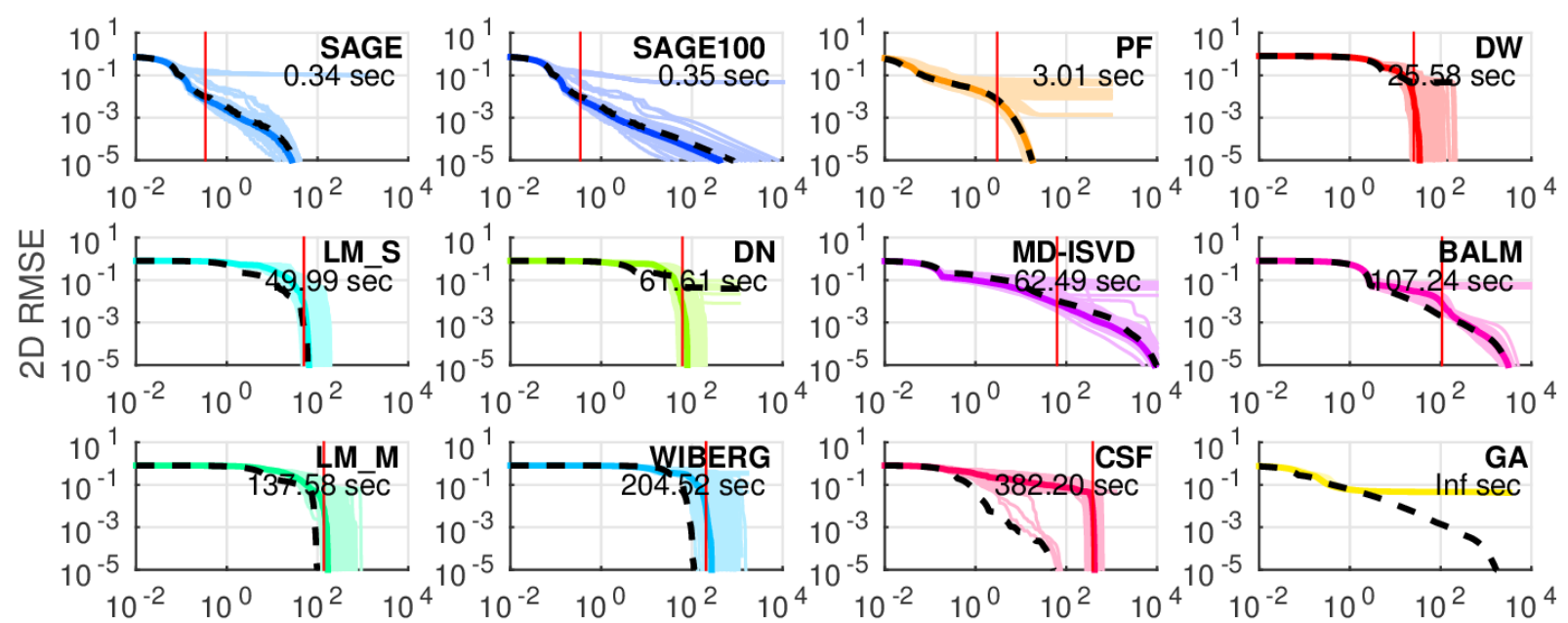

Time (s)
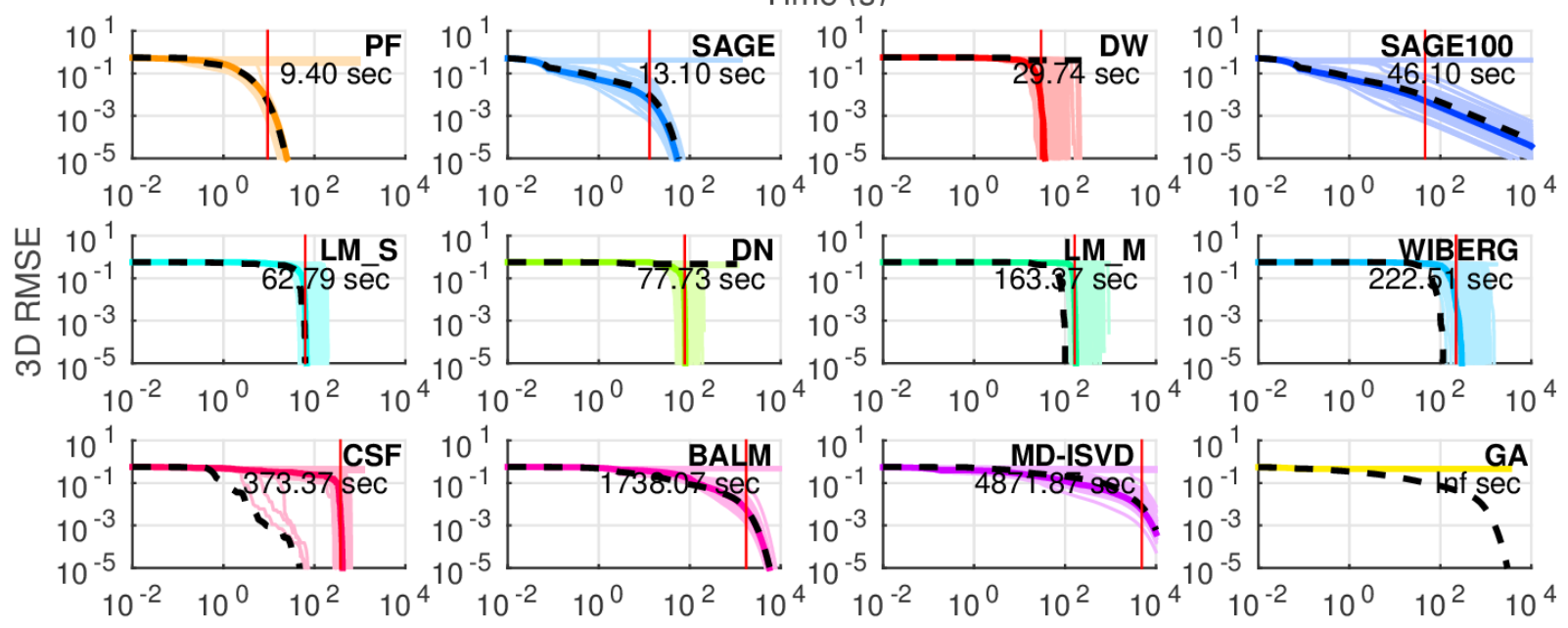

Time (s)

Figure 4: Results on the Synthetic Sphere dataset with a banded occlusion pattern. Top plots show 2D RMSE over time. All 100 random initializations are plotted in light colors, the median of which is plotted in a darker color. The result of the deterministic initialization is shown as a dashed black line. The number below each algorithm name is the time for the median run to go $99 \%$ of the way from the initial error to the minimum error. The location of this point is shown using a vertical red line and the algorithms are sorted by this number. Bottom plots show the same values, but for 3D RMSE.

outliers increases. For SFM problems containing sparse outliers, RSAGE can therefore be used to find an accurate 3D model.

We note that there are many batch approaches designed specifically for robust matrix factorization in the presence of sparse outliers [29, 31, 28. The purpose here is only to note that robustness can be added to our own model in a straightforward way. We leave a thorough comparison to robust batch methods as future work. Since we have found RSAGE to be much slower than SAGE, real-time performance demands may require us to to adjust point tracking so that only high-quality tracks are used, and run SAGE (rather than RSAGE) on this reduced data set. 


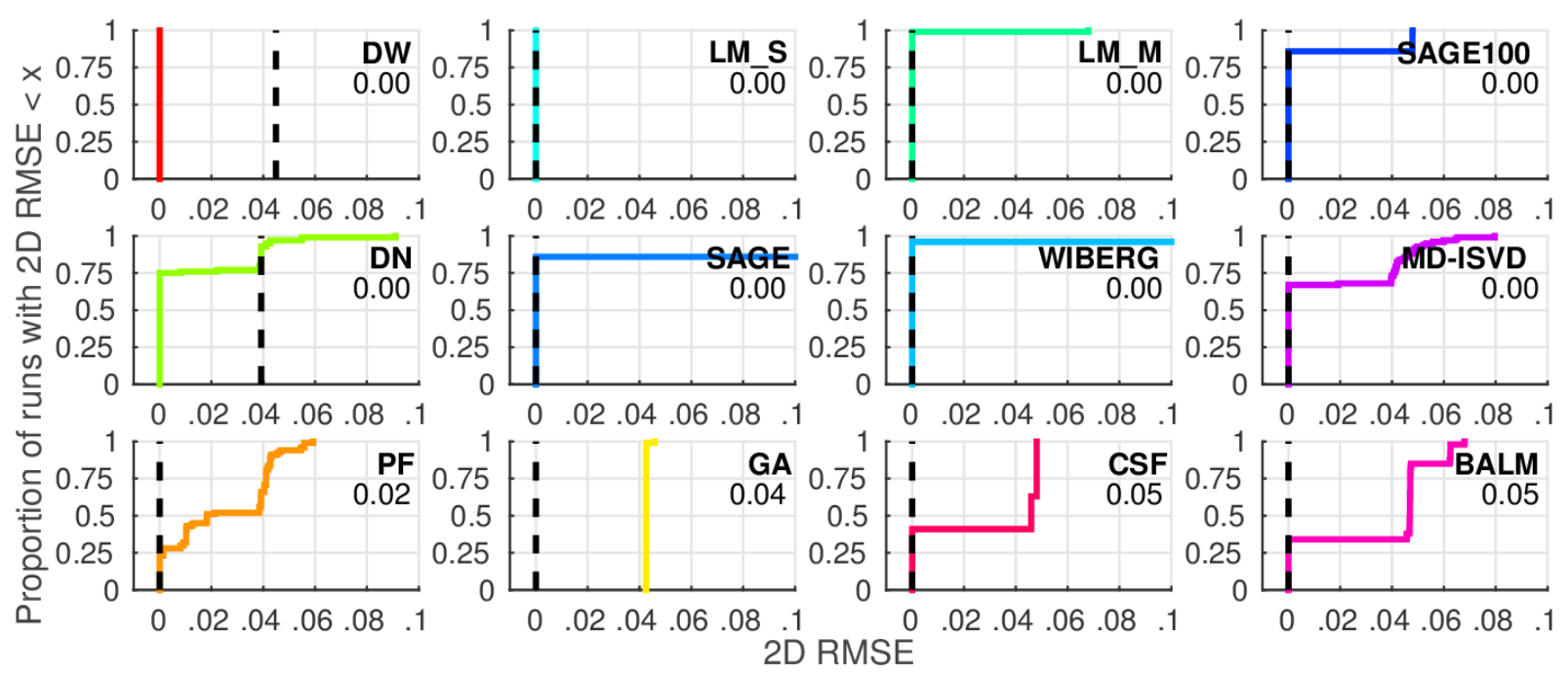

Figure 5: Empirical CDF results on the Synthetic Sphere dataset with a banded occlusion pattern. These plots show the empirical cumulative distribution of error after convergence. The error of the deterministic initialization is shown as a vertical dashed black line. The number associated with each algorithm is the median final error, and the algorithms are sorted by this value. The CDF plots for 3D RMSE are very similar and thus are not shown.
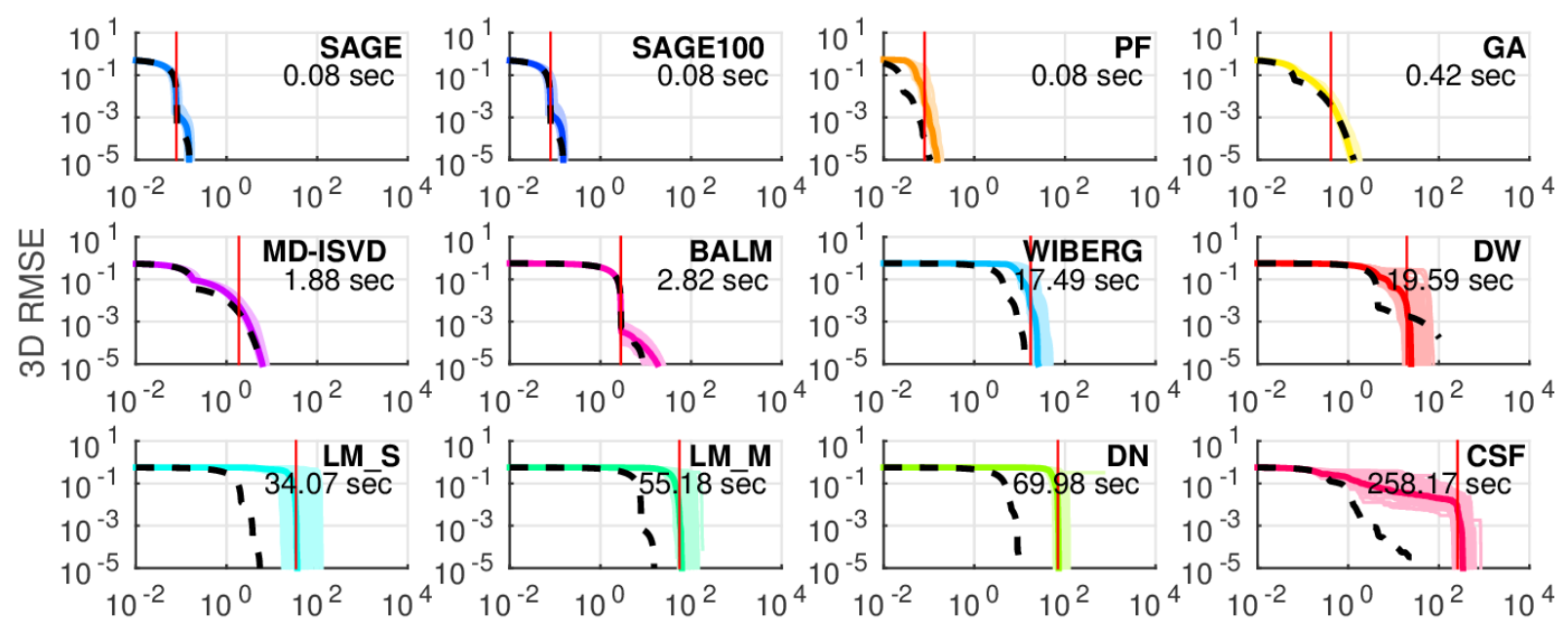

Time (s)

Figure 6: Results on the synthetic Sphere dataset with a random occlusion pattern, measured using 3D RMSE. See the text for details. All algorithms achieve nearly universal convergence on this dataset, and we find that SAGE, SAGE100 and PF are much faster than other approaches.

\subsubsection{Large dataset}

In addition to its speed, another advantage of SAGE is that it is memory efficient and can be used on very large datasets where second-order methods are much too computationally burdensome. In [41, the algorithm BALM was used to reconstruct a very large synthetic 3D model, and we compare SAGE to them using the same dataset. The dataset used is Venice from Agarwal et. al [43, as modified in [41]: the 3D model was projected onto 100 random orthographic cameras, producing a measurement matrix of size $939551 \times 200$ from which $90 \%$ of the entries were randomly removed. We measure the error as $\left\|S-S_{g t}\right\|_{F} /\left\|S_{g t}\right\|_{F}$, where 

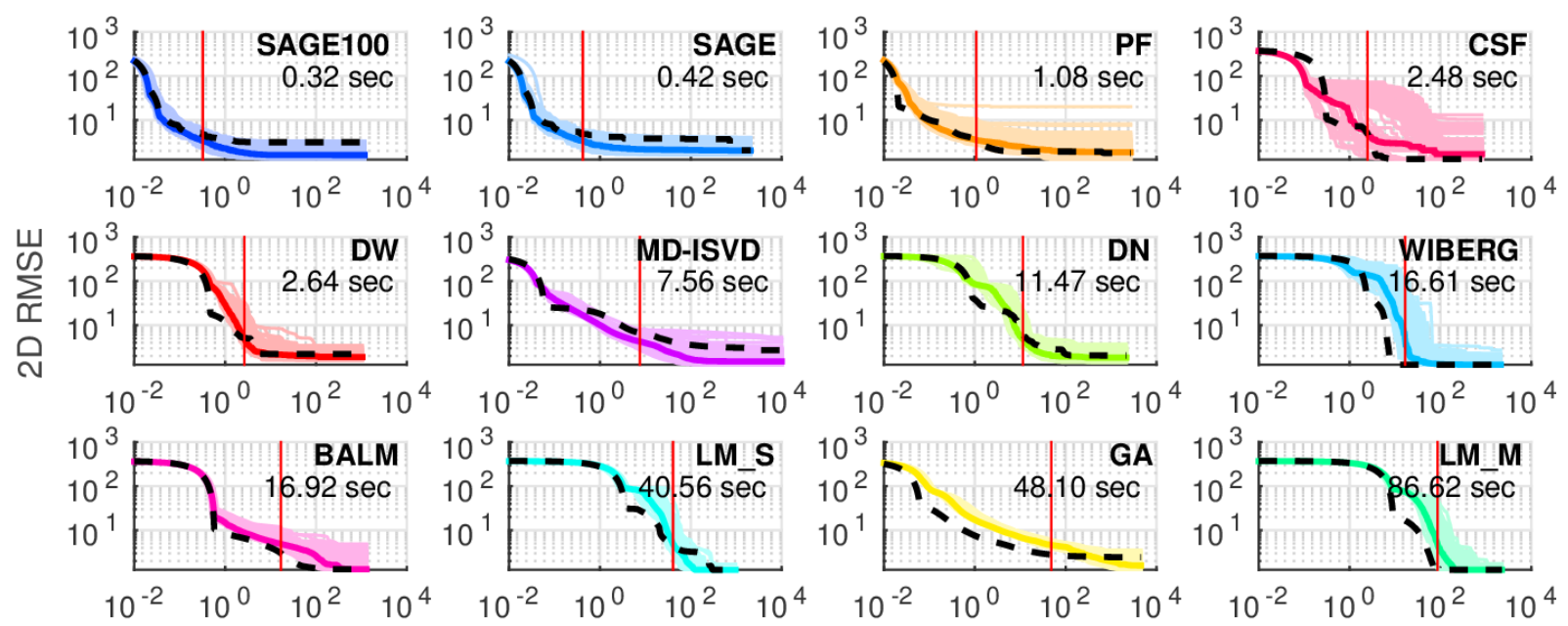

Time (s)
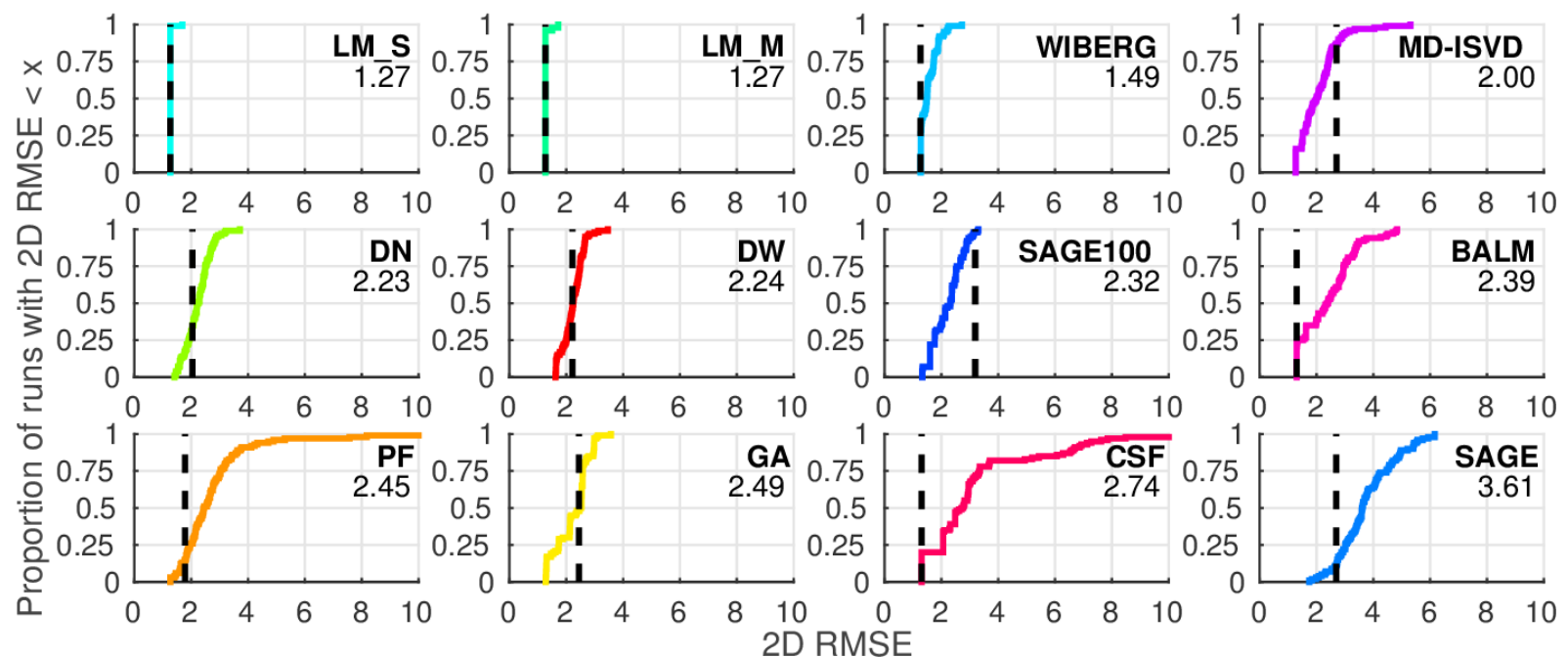

Figure 7: Result for the Dino dataset. Top plots show the convergence of algorithms over time. Bottom plots show the empirical cumulative distribution of 2D RMSE for each algorithm. In both cases, the deterministic initialization is shown using a dashed black line. See the text for details.

$S$ is the resulting 3D reconstruction and $S_{g t}$ is the ground-truth 3D model. The algorithm of [41] reported a reconstruction error of $6.67 \%$, with no computational time reported. SAGE achieved this reconstruction error within 50 seconds. SAGE then further reduced the error to $2.48 \%$ after 2 minutes, and to $1.37 \%$ after 10 minutes.

\subsection{Online experiments}

\subsubsection{Real-time implementation}

To demonstrate the use of our method in real-time reconstruction, we implemented SAGE in C++ using OpenCV. Our implementation uses two threads running on separate cores: One thread reads frames from an attached webcam and tracks point using the KLT tracker in OpenCV, while the other thread continually runs SAGE and incorporates new data as it becomes available. We used a MacBook Pro with a $2.66 \mathrm{GHz}$ Intel Core 2 Duo processor and 8 GB of memory, with a Logitech C270 webcam. 

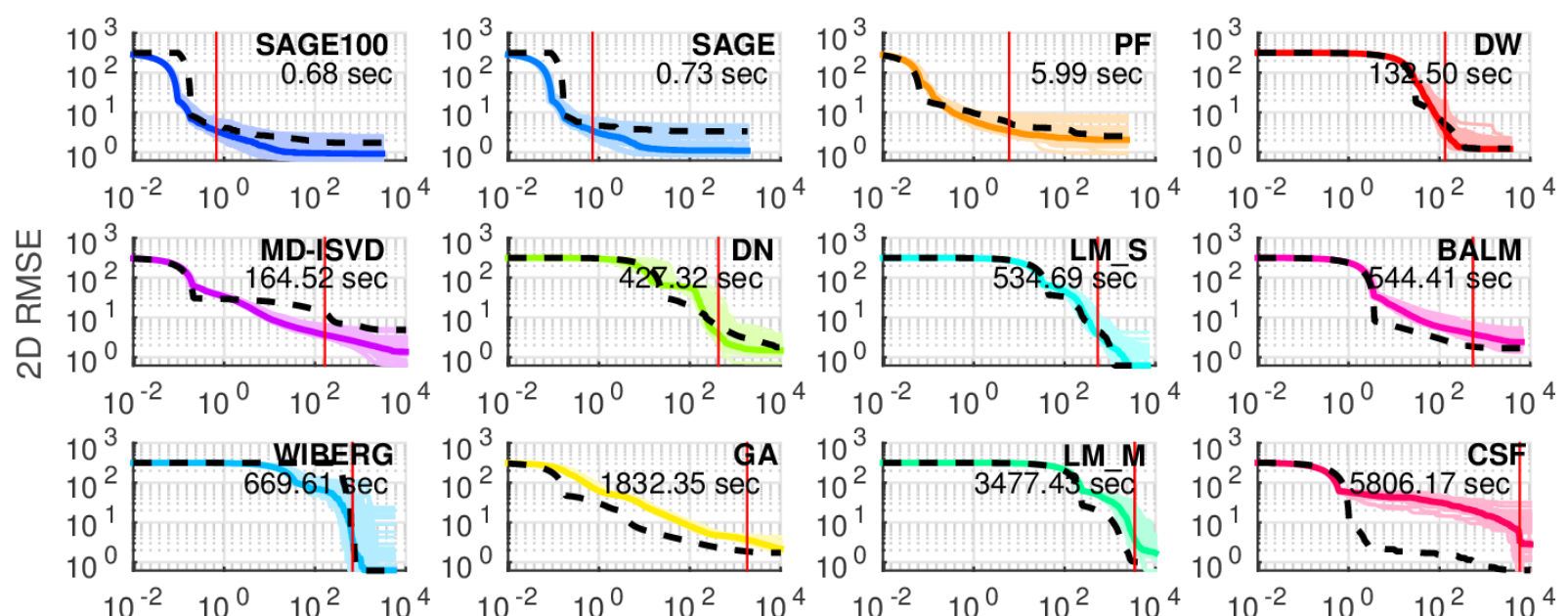

Time (s)
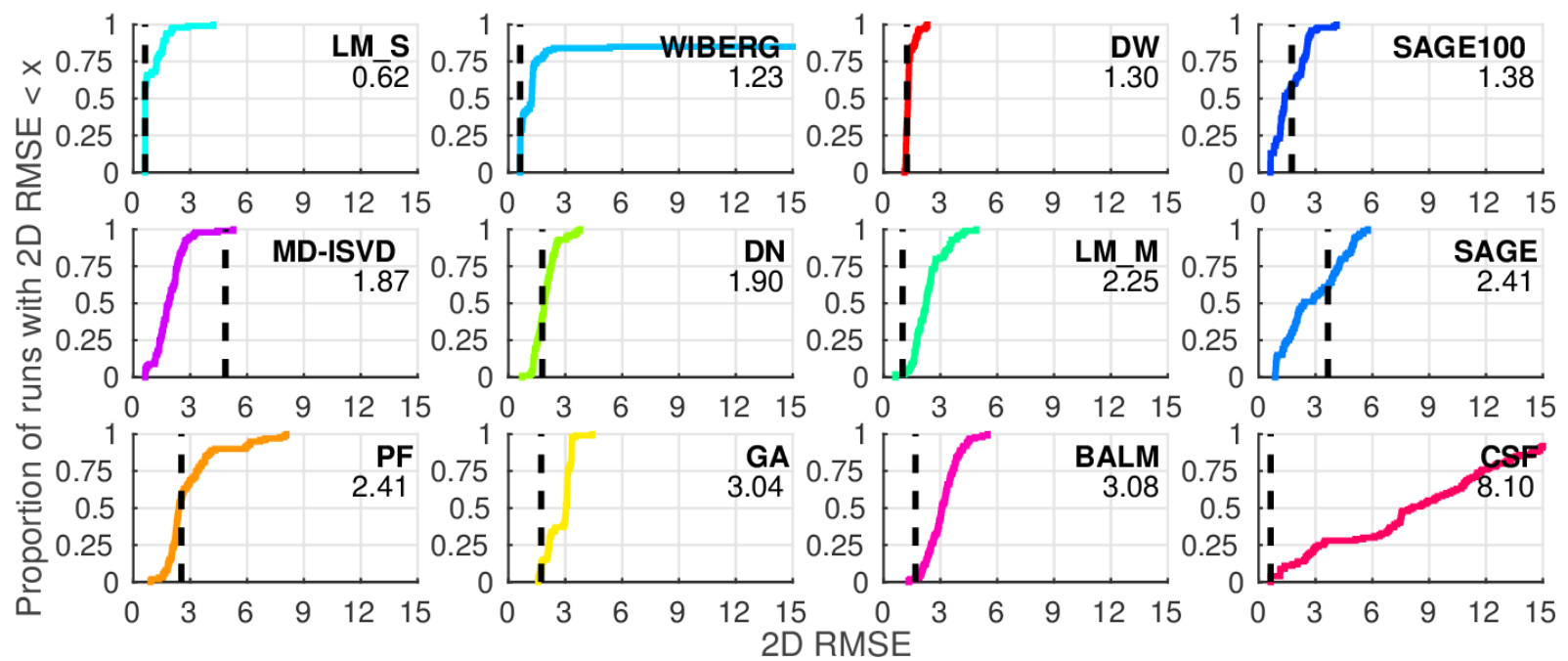

Figure 8: Result for the Bear dataset. Top plots show the convergence of algorithms over time. Bottom plots show the empirical cumulative distribution of 2D RMSE for each algorithm. In both cases, the deterministic initialization is shown using a dashed black line. See the text for details.

The implementation was run at 15 fps and we used it to capture and reconstruct a 3D model of a toy giraffe in real time (Figure 10). The bottleneck in this process is tracking points between frames, and during this time SAGE processed an average of 205 columns per frame, each column being selected randomly from the past columns of the measurement matrix. Since this approach is able to use a simple webcam and a standard computer to obtain a 3D model in real time, we envision it being useful in applications where it is desirable to build a 3D model with low-cost hardware, such as at-home 3D printing.

\subsubsection{Comparison}

In the online setting, we compare MD-ISVD to SAGE for which the residual is not scaled, as well as to SAGE100 for which the residual for a column $c$ is scaled by $C /(C+t(c))$ where $t(c)$ indicates the number of times that column $c$ has been processed. A related incremental algorithm is also given in 20. We attempted to compare to this method as well, but found that it was extremely unstable because it requires calculating the determinant of matrices with very large eigenvalues. For this reason, its results are not presented here. 


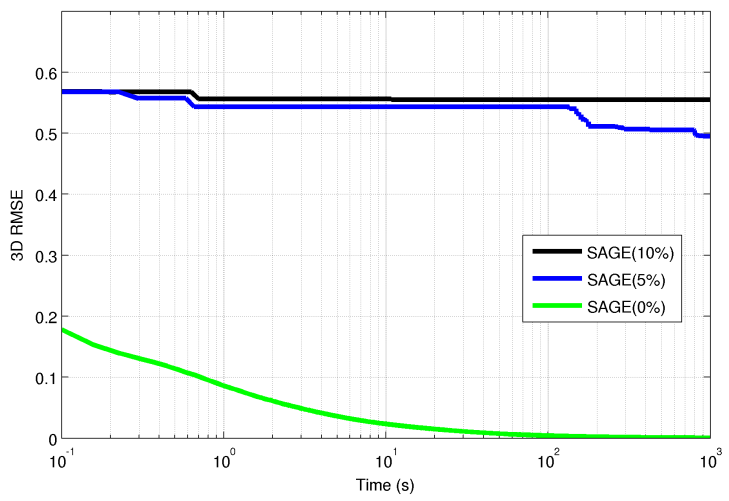

(a) SAGE

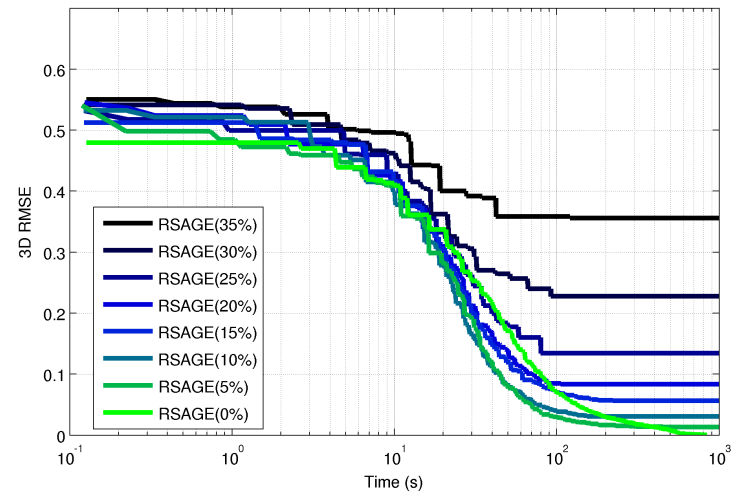

(b) RSAGE100

Figure 9: Effect of sparse noise on SAGE100 and RSAGE100 using the Synthetic Sphere dataset with a banded occlusion pattern. The algorithms have a decay factor on the residual of $\alpha_{t}=C /(C+t)$, with $C=100$. A variable proportion of the data were set to sparse outliers, from $0 \%$ to $35 \%$, and we measure the 3D RMSE over time. Even a small number of sparse outliers causes SAGE to converge to an incorrect 3D model, while the 3D RMSE decays gradually with RSAGE. When no sparse outliers are present, SAGE converges much faster than RSAGE.

Each algorithm was implemented in MATLAB for this comparison.

In contrast to the batch case, for the online experiments the frames are processed sequentially and only data up to the current frame is available. The algorithms are compared by varying the number of iterations spent processing each frame and evaluating the RMSE after the last frame.

In addition to the Sphere, Dino, and Bear sequences, we also use a new sequence of a stuffed Giraffe. This dataset was gathered in real time; the details of the real time implementation are given in Section 6.2.1. The Giraffe dataset is shown in Figure 10, and consists of 2634 points over 343 frames, with $93.4 \%$ missing data. Note that this dataset is significantly larger than the other sequences and most second-order batch algorithms would be computationally intractable due to the large number of variables.

Results for online experiments are given in Figure 11. In all cases, SAGE and SAGE100 outperform MD-ISVD over a range of framerates. In addition, we find that for the Dino sequence, the scaled algorithm SAGE100 performs somewhat better than the unscaled SAGE; the decaying step size seems to help. The opposite is observed for the Sphere dataset, where SAGE outperforms SAGE100, but in this case there is no noise and so reducing downscaling the residual only serves to reduce the convergence rate.

SAGE is able to achieve a very low RMSE in all cases using a very high framerate. For the shorter Dino sequence, it may be necessary to reduce the framerate to $5 \mathrm{fps}$ to achieve a low RMSE, but for the other sequences a high framerate can be used since the algorithm has more of an opportunity to re-visit old frames in these longer sequences. In fact, for the Bear and Giraffe sequences, a framerate of $60 \mathrm{fps}$ is sufficient for an accurate reconstruction.

For the Sphere dataset, Figure 11 also shows the resulting 3D reconstruction that is obtained after running SAGE at 15 fps. The model obtained is very close to the groundtruth, demonstrating that we are indeed able to find accurate solutions in real time.

\section{Conclusion}

In this paper we have proposed the use of SAGE and its robust counterpart RSAGE for factorizationbased structure from motion. Although there are many other approaches for this problem, they are generally either fast but with unreliable convergence ( $\mathrm{PF}$ and $\mathrm{GA}$ ) or use second-order information to improve their 


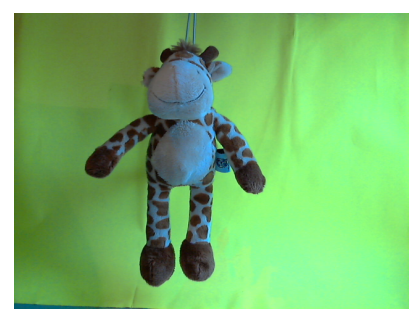

(a)

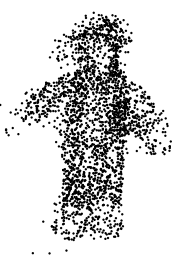

(b)

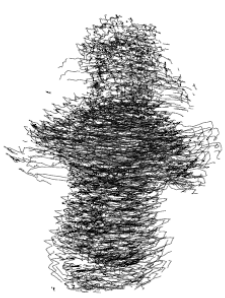

(c)

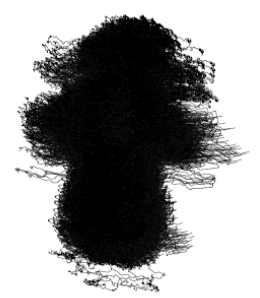

(d)

Figure 10: Giraffe dataset. (a) One frame from the sequence. (b) Reconstructed 3D model. (c) Observed point tracks. (d) Final reconstruction of point tracks after running SAGE online.

convergence but are slower (DN, LM_M and LM_S). We have demonstrated that SAGE converges quickly to solutions of good quality. SAGE performs exceptionally well on batch problems and is orders of magnitude faster than other algorithms that achieve a similar accuracy. However, we have also observed that SAGE can have difficulty reaching a highly accurate best solution, due to it being a first-order stochastic gradient algorithm. For applications where both speed an accuracy are required, SAGE could be used to rapidly find a solution of reasonable quality, which can then be used as a starting point for another algorithm that may find a more accurate final solution (such as LM_M or LM_S).

We have also shown that SAGE performs well in the online setting, where the aim is to build a 3D model of an object in real time as the video is taken. The efficiency of SAGE allows the use of low-cost hardware such as a laptop and webcam, or a cellphone. Furthermore, even if a slow-but-accurate algorithm such as LM_M or LM_S is used to obtain the final 3D model, the structure determination process is sped up by having a good initialization available from the real-time execution of SAGE.

\section{Acknowledgements}

Work on this paper by Laura Balzano was partially supported by Army Research Office grant W911NF14-1-0634. Stephen Wright acknowledges the support of NSF Awards DMS-1216318 and IIS-1447449, ONR Award N00014-13-1-0129, AFOSR Award FA9550-13-1-0138, and Subcontract 3F-30222 from Argonne National Laboratory. 


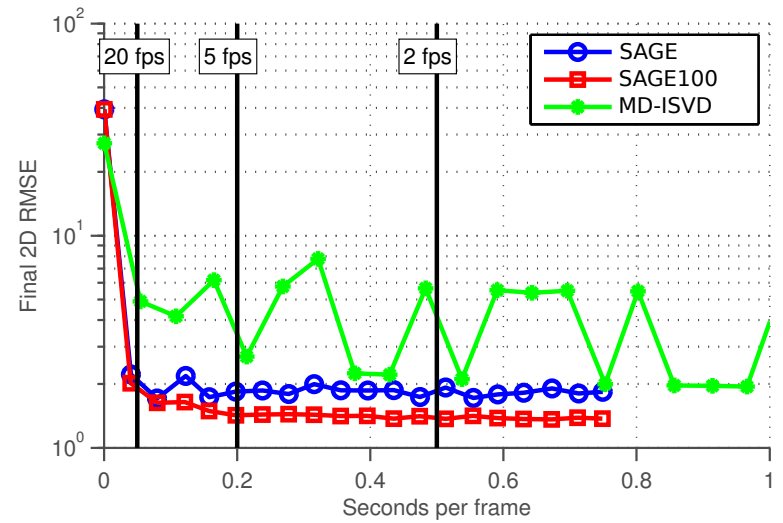

(a) Dino

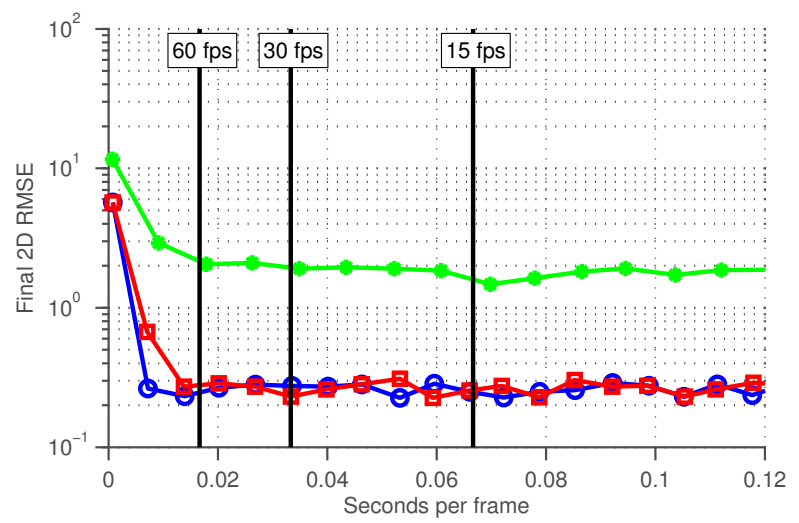

(c) Giraffe

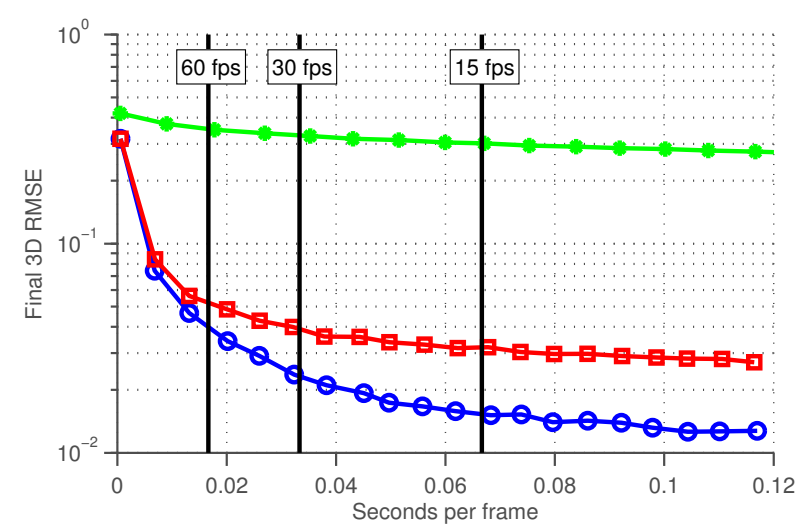

(e) Sphere (3D RMSE)

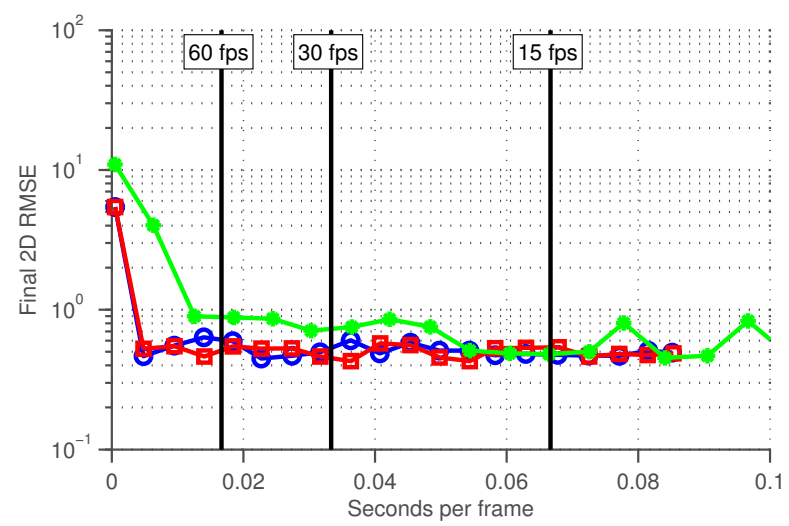

(b) Bear

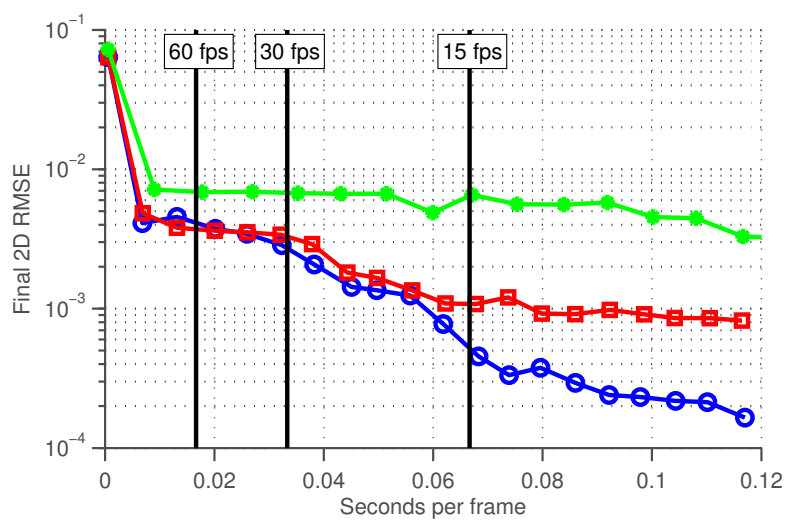

(d) Sphere (2D RMSE)

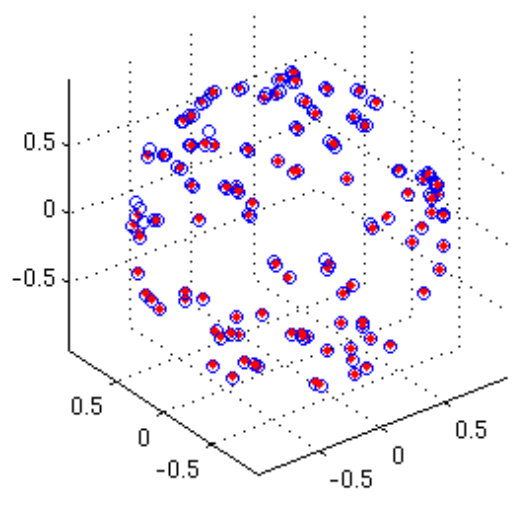

(f) Groundtruth 3D model (red) and estimated model (blue) for SAGE after running at 15 fps.

Figure 11: Online experiments. We compare MD-ISVD, SAGE and SAGE100 and vary the number of iterations spent on each frame. In all cases, SAGE and SAGE100 achieve a low RMSE even for a high framerate. For the synthetic Sphere dataset, we shown both 2D and 3D RMSE. In (f), we shown the 3D model generated using SAGE in an online setting when run at 15 frames per second on the Sphere dataset. 


\section{References}

[1] C. Tomasi, T. Kanade, Shape and motion from image streams under orthography: a factorization method, International Journal of Computer Vision 9 (2) (1992) 137-154.

[2] E. Candès, T. Tao, The power of convex relaxation: Near-optimal matrix completion, IEEE Transactions on Information Theory 56 (5) (2010) $2053-2080$.

[3] B. Recht, A simpler approach to matrix completion, Journal of Machine Learning Research 12 (2011) $3413-3430$.

[4] E. J. Candès, X. Li, Y. Ma, J. Wright, Robust principal component analysis?, Journal of the ACM 58 (1) (2009) 1-37.

[5] E. Candes, Y. Plan, Matrix completion with noise, Proceedings of the IEEE 98 (6) (2010) 925-936.

[6] L. Balzano, R. Nowak, B. Recht, Online identification and tracking of subspaces from highly incomplete information, in: Communication, Control, and Computing (Allerton), IEEE, 2010, pp. 704-711.

[7] L. Balzano, S. Wright, Local convergence of an algorithm for subspace identification from partial data, Foundations of Computational Mathematics (2014) 1-36doi:10.1007/s10208-014-9227-7. URL http://dx.doi.org/10.1007/s10208-014-9227-7

[8] J. He, L. Balzano, A. Szlam, Incremental gradient on the grassmannian for online foreground and background separation in subsampled video, in: Computer Vision and Pattern Recognition, 2012.

[9] C. Poelman, T. Kanade, A paraperspective factorization method for shape and motion recovery, Pattern Analysis and Machine Intelligence 19 (3) (1997) 206-218.

[10] H. Aanæs, R. Fisker, K. Astrom, J. Carstensen, Robust factorization, Pattern Analysis and Machine Intelligence 24 (9) (2002) 1215-1225.

[11] A. Buchanan, A. Fitzgibbon, Damped newton algorithms for matrix factorization with missing data, in: Computer Vision and Pattern Recognition (CVPR), Vol. 2, IEEE, 2005, pp. 316-322.

[12] P. Gotardo, A. Martinez, Computing smooth time trajectories for camera and deformable shape in structure from motion with occlusion, Pattern Analysis and Machine Intelligence 33 (10) (2011) 20512065.

[13] R. Guerreiro, P. Aguiar, 3d structure from video streams with partially overlapping images, in: International Conference on Image Processing, Vol. 3, IEEE, 2002, pp. 897-900.

[14] T. Morita, T. Kanade, A sequential factorization method for recovering shape and motion from image streams, Pattern Analysis and Machine Intelligence 19 (8) (1997) 858-867.

[15] J. Tardif, A. Bartoli, M. Trudeau, N. Guilbert, S. Roy, Algorithms for batch matrix factorization with application to structure-from-motion, in: Computer Vision and Pattern Recognition, IEEE, 2007, pp. $1-8$.

[16] G. Klein, D. Murray, Parallel tracking and mapping for small ar workspaces, in: International Symposium on Mixed and Augmented Reality, IEEE, 2007, pp. 225-234.

[17] E. Mouragnon, M. Lhuillier, M. Dhome, F. Dekeyser, P. Sayd, Generic and real-time structure from motion using local bundle adjustment, Image and Vision Computing 27 (8) (2009) 1178-1193.

[18] P. McLauchlan, D. Murray, A unifying framework for structure and motion recovery from image sequences, in: International Conference on Computer Vision, IEEE, 1995, pp. 314-320. 
[19] M. Trajković, M. Hedley, A practical algorithm for structure and motion recovery from long sequence of images, in: Image Analysis and Processing, Springer, 1997, pp. 470-477.

[20] R. Cabral, J. Costeira, F. De la Torre, A. Bernardino, Fast incremental method for matrix completion: an application to trajectory correction, in: International Conference on Image Processing, IEEE, 2011, pp. $1417-1420$.

[21] J. R. Bunch, C. P. Nielsen, Updating the singular value decomposition, Numerische Mathematik 31 (1978) 111-129, 10.1007/BF01397471.

URL http://dx.doi.org/10.1007/BF01397471

[22] M. Brand, Incremental singular value decomposition of uncertain data with missing values, European Conference on Computer Vision (2002) 707-720.

[23] E. Candès, B. Recht, Exact matrix completion via convex optimization, Foundations of Computational Mathematics 9 (6) (2009) 717-772.

[24] R. Keshavan, A. Montanari, S. Oh, Matrix completion from noisy entries, Journal of Machine Learning Research 11 (2010) 2057-2078.

[25] K.-C. Toh, S. Yun, An accelerated proximal gradient algorithm for nuclear norm regularized least squares problems, Pacific Journal of Optimization 6 (2010) 615-640.

[26] L. Balzano, Handling missing data in high-dimensional subspace modeling, Ph.D. thesis, University of Wisconsin, Madison (2012).

[27] A. Edelman, T. A. Arias, S. T. Smith, The geometry of algorithms with orthogonality constraints, SIAM Journal on Matrix Analysis and Applications 20 (2) (1998) 303-353.

[28] D. Strelow, General and nested wiberg minimization, in: Computer Vision and Pattern Recognition (CVPR), 2012 IEEE Conference on, IEEE, 2012, pp. 1584-1591.

[29] A. Eriksson, A. Van Den Hengel, Efficient computation of robust low-rank matrix approximations in the presence of missing data using the 11 norm, in: Computer Vision and Pattern Recognition (CVPR), 2010 IEEE Conference on, IEEE, 2010, pp. 771-778.

[30] N. Wang, T. Yao, J. Wang, D.-Y. Yeung, A probabilistic approach to robust matrix factorization, in: Computer Vision-ECCV 2012, Springer, 2012, pp. 126-139.

[31] Y. Zheng, G. Liu, S. Sugimoto, S. Yan, M. Okutomi, Practical low-rank matrix approximation under robust 1 1-norm, in: Computer Vision and Pattern Recognition (CVPR), 2012 IEEE Conference on, IEEE, 2012, pp. 1410-1417.

[32] G. Mateos, G. B. Giannakis, Sparsity control for robust principal component analysis, in: Asilomar Conference on Signals, Systems, and Computers, 2010.

[33] J. He, L. Balzano, J. Lui, Online robust subspace tracking from partial information, Arxiv preprint arXiv:1109.3827

[34] M. Brand, Fast low-rank modifications of the thin singular value decomposition, Linear algebra and its applications 415 (1) (2006) 20-30.

[35] L. Balzano, S. J. Wright, On grouse and incremental svd, Workshop on Computational Advances in Multi-Sensor Adaptive Processing (CAMSAP).

[36] S. Boyd, N. Parikh, E. Chu, B. Peleato, J. Eckstein, Distributed optimization and statistical learning via the alternating direction method of multipliers, Foundations and Trends in Machine Learning 3 (1) (2011) 1-123. 
[37] P. Chen, Optimization algorithms on subspaces: Revisiting missing data problem in low-rank matrix, International Journal of Computer Vision 80 (1) (2008) 125-142.

[38] R. Hartley, F. Schaffalitzky, Powerfactorization: 3d reconstruction with missing or uncertain data, in: Australia-Japan Advanced Workshop on Computer Vision, Vol. 74, 2003, pp. 76-85.

[39] T. Okatani, K. Deguchi, On the wiberg algorithm for matrix factorization in the presence of missing components, International Journal of Computer Vision 72 (3) (2007) 329-337.

[40] T. Okatani, T. Yoshida, K. Deguchi, Efficient algorithm for low-rank matrix factorization with missing components and performance comparison of latest algorithms, in: Computer Vision (ICCV), 2011 IEEE International Conference on, IEEE, 2011, pp. 842-849.

[41] A. Del Bue, J. Xavier, L. Agapito, M. Paladini, Bilinear modeling via augmented lagrange multipliers (balm), Pattern Analysis and Machine Intelligence 34 (8) (2012) 1496-1508.

[42] A. Fitzgibbon, G. Cross, A. Zisserman, Automatic 3d model construction for turn-table sequences, 3D Structure from Multiple Images of Large-Scale Environments (1998) 155-170.

[43] S. Agarwal, N. Snavely, S. M. Seitz, R. Szeliski, Bundle adjustment in the large, in: European Conference on Computer Vision, Springer, 2010, pp. 29-42. 